\title{
Optimization of Footbridges Composed of Prismatic Tensegrity Modules
}

\author{
Jonas Feron'; Lindy Boucher²; Vincent Denoël ${ }^{3}$; and Pierre Latteur ${ }^{4}$
}

\begin{abstract}
The architectural potential of tensegrity structures is proven. Yet, paradoxically, very few real construction projects have been built around the world. The main reasons are complex construction processes, lack of design and optimization guidelines, and excessive self-weight due to the prestress needed to guarantee stiffness and dynamic behavior. Hence, optimizing the stiffness and self-weight is a key aspect when designing a tensegrity footbridge. Previous research has demonstrated the validity of a design and optimization methodology, based on morphological indicators, that identifies geometry with a maximum stiffness and/or a minimum self-weight for a family of structures. In this paper, that methodology is applied to footbridges composed of tensegrity modules comprising simplex, quadruplex, pentaplex, and hexaplex types. A comparison of the stiffest and lightest structures is provided, a practical case study is developed, and the relevance and feasibility of such tensegrity footbridges are discussed. As a result, the study provides advice on optimum footbridge topologies with the following characteristics: excellent stiffness and dynamic behavior; efficient structures composed of simplex modules; and self-weight that is still rather high but similar to that of bended structures, although with potential to be reduced thanks to optimization of the prestress scenario. DOI: 10.1061/(ASCE)BE.1943-5592.0001438. (c) 2019 American Society of Civil Engineers.
\end{abstract}

Author keywords: Tensegrity; Footbridge; Optimization; Feasibility; Stiffness; Volume; Mass; Self-weight; Morphological indicators.

\section{State of the Art and Objectives}

Tensegrity is a word with controversial definitions (Hanaor 2012). The term tensegrity system often refers to a system in a stable selfequilibrated state comprising a discontinuous set of compressed components inside a continuum of tensioned components (Motro 2005). In order to widen the potential of tensegrity to engineering applications, it is also often considered that a tensegrity structure may have $k$ compressive components in contact on a single node. In this case, Skelton and de Oliveira (2009, pp. 1-7) wrote about a structure "of class $k$." Extended tensegrity refers, in this article, to other definitions of tensegrity. This includes, among others, structures with some members subjected to bending moments or structures that are prestressable only if supports exist.

${ }^{1}$ Ph.D. Student, Univ. catholique de Louvain, Louvain School of Engineering EPL, Civil and Environmental Engineering, Place du Levant, 1 (Vinci), bte L5.05.01, 1348-Louvain-la-Neuve, Belgium; BESIX, Avenue des Communautés, 100, 1200 Bruxelles, Belgium. Email: jonas.feron@ uclouvain.be; jferon@besix.com

${ }^{2}$ Master's Student, Univ. catholique de Louvain, Louvain School of Engineering EPL, Civil and Environmental Engineering, Place du Levant, 1 (Vinci), bte L5.05.01, 1348-Louvain-la-Neuve, Belgium. ORCID: https:// orcid.org/0000-0002-3342-1800. Email: lindy-boucher@ hotmail.com

${ }^{3}$ Professor, Univ. de Liège, Faculté des Sciences Appliquées, Argenco, Quartier Polytech 1, Allée de la Découverte, 9, 4000 Liège, Belgium. ORCID: https://orcid.org/0000-0002-7256-1734. Email: v.denoel@ulg.ac.be

${ }^{4}$ Professor, Univ. catholique de Louvain, Louvain School of Engineering EPL, Civil and Environmental Engineering, Place du Levant, 1 (Vinci), bte L5.05.01, 1348-Louvain-la-Neuve, Belgium (corresponding author). ORCID: https://orcid.org/0000-0002-4608-3732. Email: pierre .latteur@uclouvain.be

Note. This manuscript was submitted on May 10, 2018; approved on February 08, 2019; published online on September 17, 2019. Discussion period open until February 17, 2020; separate discussions must be submitted for individual papers. This paper is part of the Journal of Bridge Engineering, (C) ASCE, ISSN 1084-0702.
Although tensegrity and extended tensegrity structures have found a significant place in scientific papers these last decades, the topic of tensegrity applied to footbridges is relatively absent in the literature. Gómez-Jáuregui (2010) established a large state of the art about tensegrity structures and their application to architecture. He cited some authors suggesting the use of tensegrity for bridge applications, but his investigations did not reveal any existence of tensegrity-based bridge proposals before 2004 . He also cited some authors describing structures having, according to them, a high stiffness and economical design. Other cited authors, on the contrary, were more skeptical about the performances of tensegrity structures in comparison with more classical structures. Yet nowadays there is still a lack of rigorous and quantitative proofs about the structural efficiency or inefficiency of tensegrity structures for engineering applications, in particular bridges and footbridges.

Fig. 1 shows a few creative prototypes with high architectural potential, which, however, never reached the construction stage.

- In his 2004 master's thesis, Gómez-Jáuregui (2010) proposed a Class 1 (no struts in contact with each other) tensegrity footbridge concept based on a succession of simplex modules [Fig. 1(a)].

- Architects from the firm WilkinsonEyre (2004) and the structural engineer C. Balmond from Arup developed a prototype of a 35$\mathrm{m}$-long tensegrity bridge composed of a mesh of tetrahedral cells [Fig. 1(b)]. The footbridge was designed to span the Great Hall of the National Building Museum in Washington, DC, and to react to pedestrians by lighting the glass struts as they were subjected to changing stresses (Davey and Forster 2007).

- Micheletti et al. (2005) presented the Tor Vergata footbridge [Fig. 1(c)], a 32-m-long Class 2 tensegrity footbridge composed of reexpanded octahedron modules. The height $(3.6 \mathrm{~m})$, the number of modules (five), and the sections of the struts $(193 \mathrm{~mm}$ diameter, $12 \mathrm{~mm}$ thick) and of the cables $(72 \mathrm{~mm})$ were chosen in order to find a compromise between maximum stiffness (deflection of $8 \mathrm{~cm}$ under the most critical static loads combination) and cost efficiency (Micheletti 2012). 


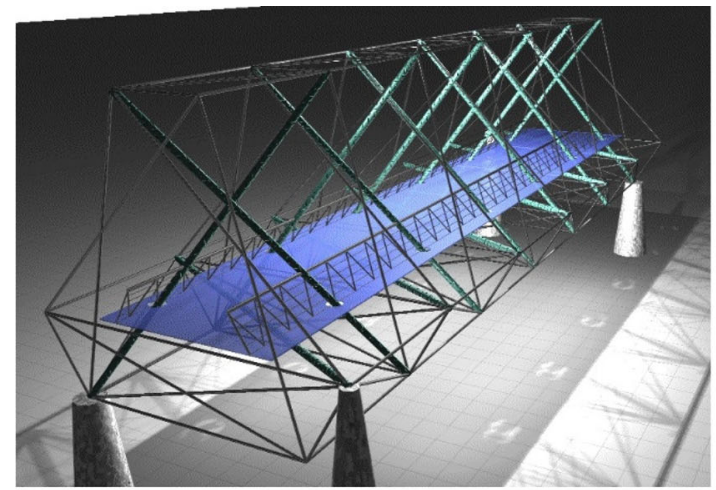

(a)

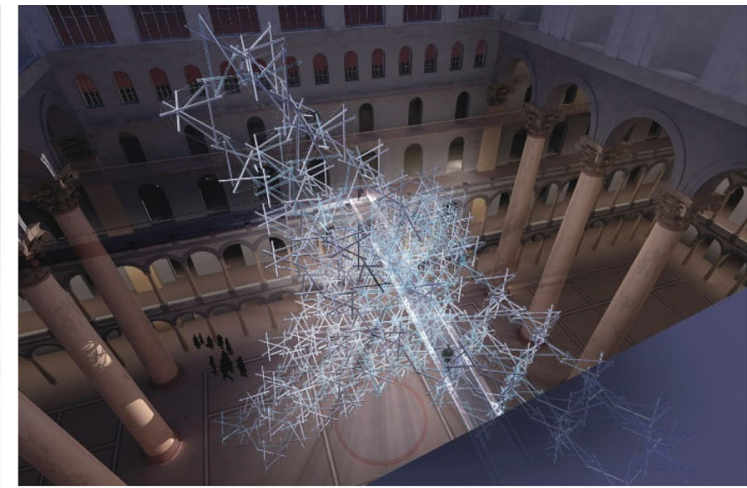

(b)

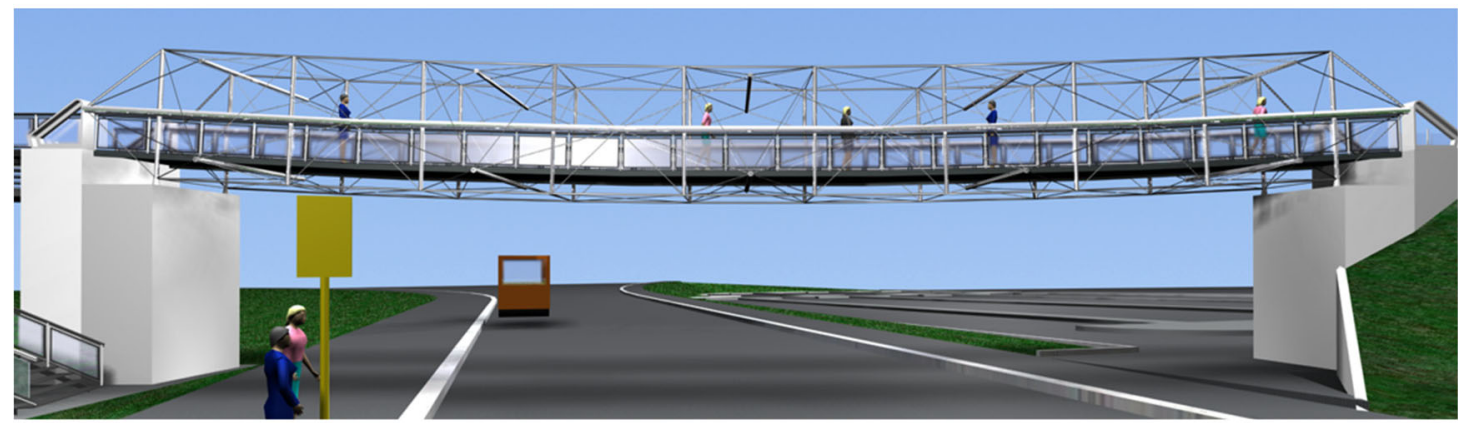

(c)
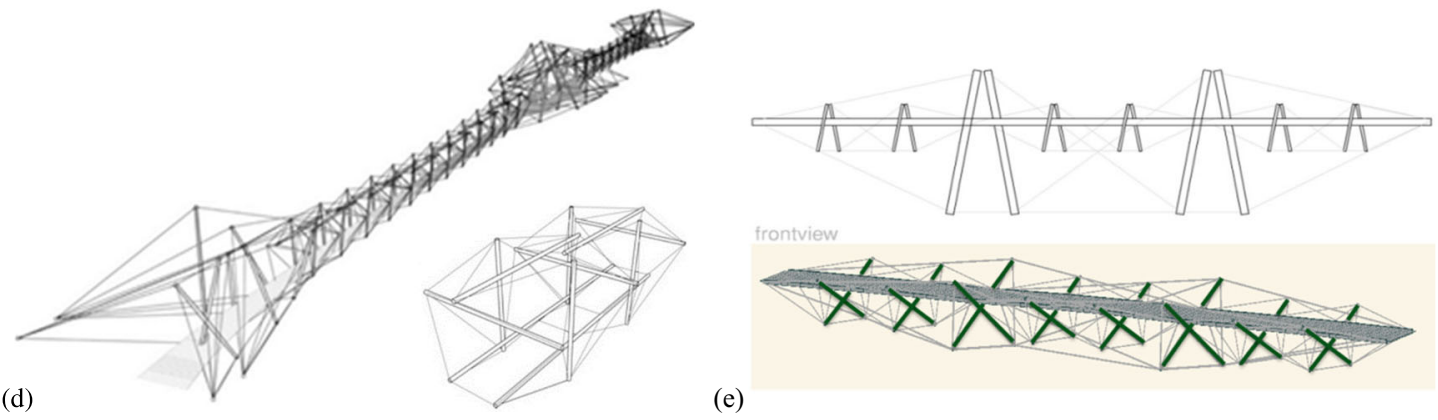

(e)

Fig. 1. Architectural projects: (a) Gómez-Jáuregui prototype (reprinted from Gómez-Jáuregui 2010, with permission); (b) WilkinsonEyre prototype (image courtesy of WilkinsonEyre); (c) Tor Vergata footbridge (image courtesy of A. Micheletti); and (d) suspended tensegrity bridge (reprinted from Mucedola and Paradiso 2013, with permission); (e) De Boeck (2013) prototype (reprinted from De Boeck 2013, with permission).

- Mucedola and Paradiso (2013) proposed a suspended tensegrity bridge project [Fig. 1(d)] intended to cross the Sesia River in Italy for the sake of the 5,900-km bicycle route of the European facilities program EuroVelo 8. The extended tensegrity footbridge, based on a reviewed reexpanded octahedron module, would consist of two main spans of $117 \mathrm{~m}$ and $78 \mathrm{~m}$, with a height of $7 \mathrm{~m}$ (headroom of $2.85 \mathrm{~m}$ ) and a straight 3.1-m-wide deck. The struts would have a diameter of $300 \mathrm{~mm}$ with a thickness of $10 \mathrm{~mm}$, while the cables would have a diameter of $40 \mathrm{~mm}$ (Mucedola and Paradiso 2013).

- De Boeck (2013) presented an extended tensegrity topology based on a succession of simplex modules according to a fractal design [Fig. 1(e)]. In each simplex module, one strut is horizontal and supports the deck. He detailed a 30-m-span structure weighing $270 \mathrm{kN}$ and able to support a distributed load of 6.25 $\mathrm{kN} / \mathrm{m}$ with a maximum deflection of $55 \mathrm{~mm}$.

In contrast, Fig. 2 shows two rare examples of existing footbridges based on the tensegrity principle.

- In 2000, the architect den Hollander (Eekhout 2016, pp. 167178) designed the Bridge of Masts in Purmerend, Netherlands. This 144-m-long pedestrian bridge in extended tensegrity is supported by two rows of sixteen masts and sixty-four tensile rods in such a way that the 3.5-m-wide deck is suspended and visually independent of the masts [Fig. 2(a)].

- In 2009, Cox Rayner Architects and Arup designed the Kurilpa Bridge in Brisbane, Australia. Rolvink et al. (2010) from Arup described this 470-m-long (main span of $120 \mathrm{~m}$ ) pedestrian bridge [Fig. 2(b)] as a multimast cable-stay structure inspired by the principle of tensegrity, which is "both lightweight and incredibly strong."

Finding the feasibility range of tensegrity (foot)bridges on the basis of structural performances such as stiffness and self-weight remains a complex optimization problem due to the requirement of nonlinear calculations combined with the large number of parameters that characterize such structures, for example, topology, span, width, height, dimensions of the cross sections, materials properties, prestress, and external loads.

Fig. 3 shows different bridge designs using the tensegrity principle that have been optimized according to different methodologies.

- Briseghella et al. (2010) studied the influence of structural height, prestress, and dimensions of the slab, beams, and cables 

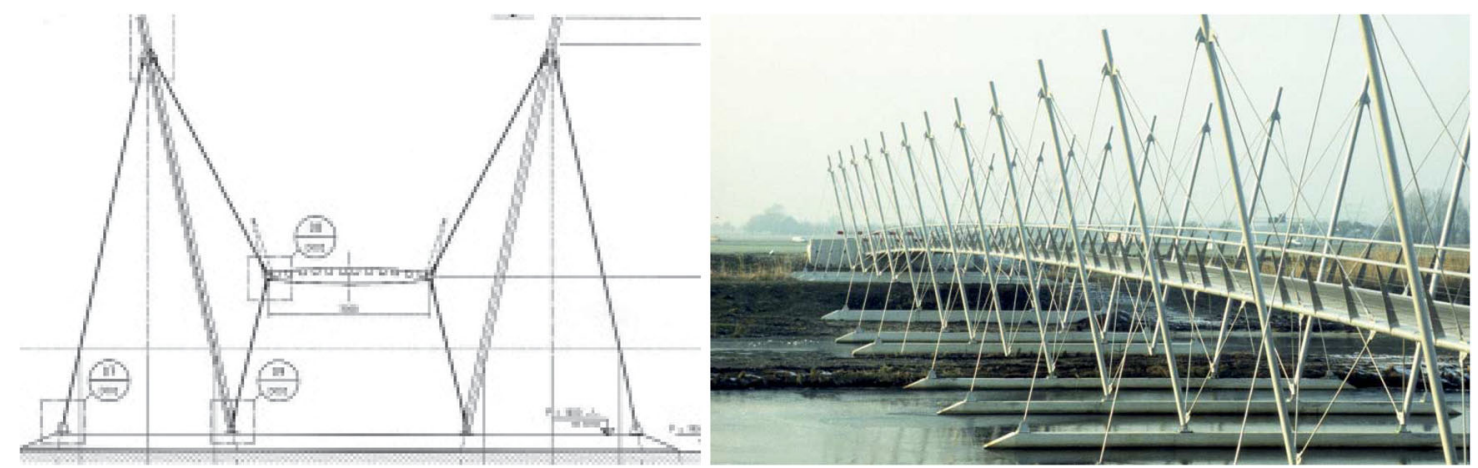

(a)

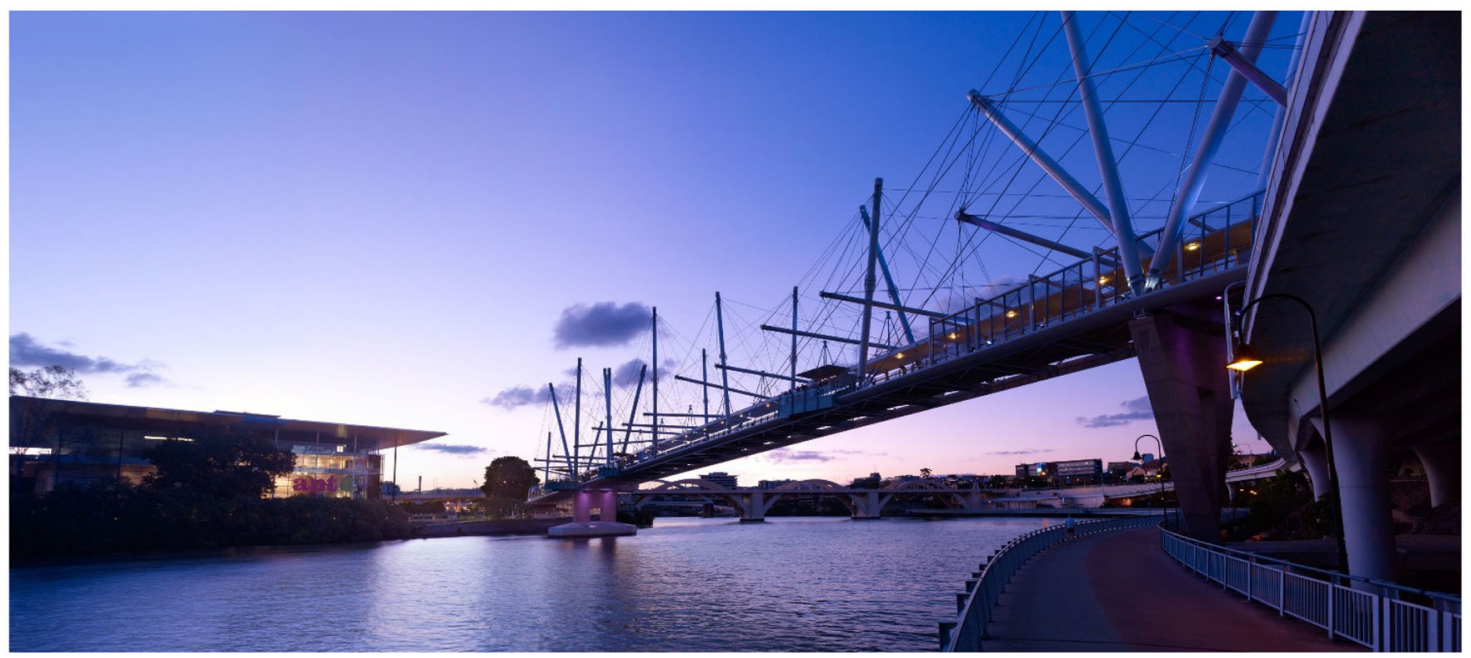

(b)

Fig. 2. Existing footbridges: (a) Bridge of Masts (2000, Purmerend, Netherlands) by den Hollander (reprinted from Eekhout 2016, with permission); and (b) Kurilpa Bridge (2009, Brisbane, Australia) by Cox Rayner Architects and Arup (Image credit: redbrickstock.com/Alamy Stock Photo).

on the dynamic behavior of a 30-m-long footbridge [Fig. 3(a)]. The prestressed arch deck is supported by four transverse rectangular beams $(500 \times 200 \times 10 \mathrm{~mm})$, which, together, are part of the extended tensegrity system. The pretensioned steel struts $(25 \mathrm{~mm}, 1,000 \mathrm{MPa})$ provide the stiffness.

- From 2010 on, several authors made significant contributions in the design of footbridges composed of tensegrity modules. They studied the influence of several parameters on structural behavior (Rhode-Barbarigos et al. 2010a; Rhode-Barbarigos et al. 2010b); analyzed dynamic behavior (Bel Hadj Ali et al. 2010); controlled deployment numerically and experimentally (Rhode-Barbarigos et al. 2012; Veuve et al. 2015; Sychterz and Smith 2017); and presented a feasible configuration of a $20-\mathrm{m}-$ span Class 4 tensegrity pedestrian bridge initially proposed in Motro et al. (2006) [Fig. 3(c)]. In particular, Rhode-Barbarigos et al. (2010a) described a design/optimization process based on parametric analysis of the cross-sectional area of the struts and cables, the rigidity ratio (cables/struts), and the self-stress level. Their method allows comparison of different modules' topologies (square, pentagonal, hexagonal) thanks to a structural efficiency index that takes into account both self-weight and deflection of the structure.

- Averseng and Dubé (2012) compared the performances, in terms of mass and deflection, of different geometries of a Class 2 deployable 12-m tensegrity beam composed of a succession of quadruplex modules. Their optimization methodology consisted of progressively increasing the prestress level and cross-sectional area until both no slacked cable criterion and allowable stresses were respected. The optimal tensegrity beam maximizing their performances indicator (defined as the inverse of the product of the deflection and the mass) showed high deflection $(L / 130)$ and important mass compared with traditional beams. Amouri et al. (2015) improved their algorithm and presented three curved tensegrity beams with three different geometries and various materials [Fig. 3(b)].

- Skelton et al. (2014) and Carpentieri et al. (2015) found the minimum mass design of a simply supported two-dimensional (2D) tensegrity bridge carrying distributed loads [Fig. 3(d)]. They used the fractal principle through repetition of the minimal mass module of Michell (1904) [Fig. 3(e)]. Their methodology consisted of finding the analytic expression of a dimensionless mass indicator in function of the topology and to minimize it under material yielding or buckling constraint.

- Finally, Latteur et al. (2017) presented a design methodology based on a stiffness and volume/mass optimization algorithm for three-dimensional (3D) nonlinear hyperstatic and prestressed structures composed of elements subjected only to axial forces, with a special emphasis on tensegrity structures. The methodology is based on dimensionless numbers called morphological indicators that allow reducing the number of parameters to consider for the stiffness and mass optimization process. It allows rigorous determination of the best topologies within a given family of structures (the best ratio of span over height, or $L / H$, and the best number of tensegrity mod- 


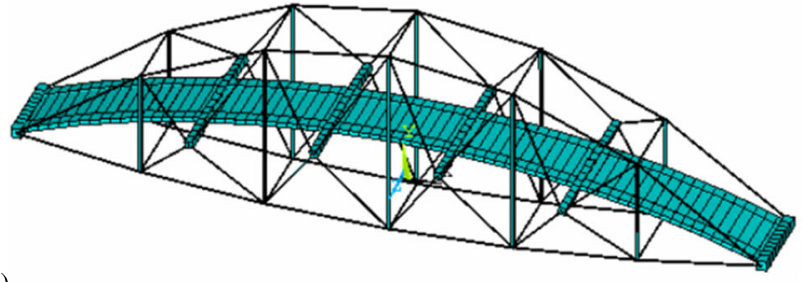

(a)

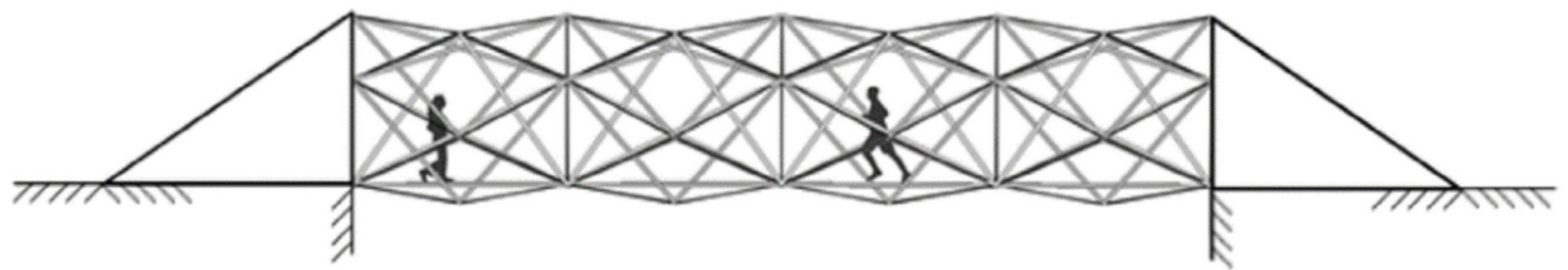

$120 \times 1 \times 2 \times$ $120 \times 1 \times+1$

(b)

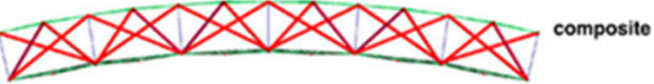

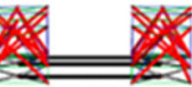

(c)

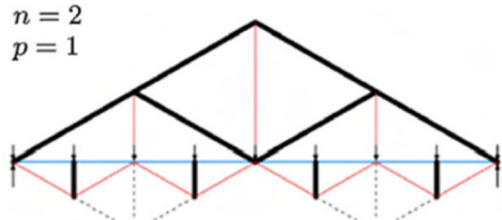

(d)

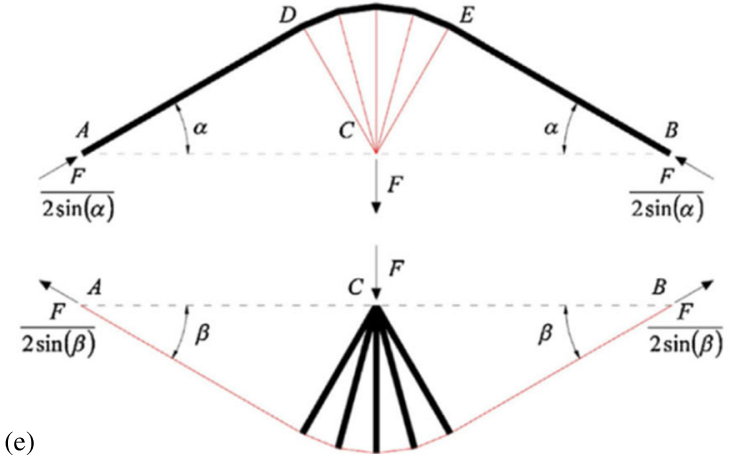

(e)

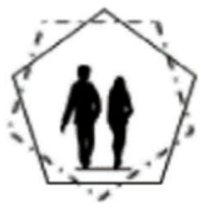

Fig. 3. Optimized bridge projects using the tensegrity principle: (a) tensegrity bridge with a prestressed deck (reprinted from Briseghella et al. 2010, with permission); (b) curved tensegrity beams (reprinted from Amouri et al. 2015, with permission from Taylor \& Francis, http://www.tandfonline.com); (c) hollow-rope footbridge (2006) (reprinted from Engineering Structures, Vol. 32, L. Rhode-Barbarigos, N. Bel Hadj Ali, R. Motro, and I. F. C. Smith, "Designing tensegrity modules for pedestrian bridges," pp. 1158-1167, (C) 2010, with permission from Elsevier); (d) 2D tensegrity bridge carrying distributed loads; and (e) Michell's minimal mass module. [Reprinted (e) and (f) from Mechanics Research Communications, Vol. 58, R. E. Skelton, F. Fraternali, G. Carpentieri, and A. Micheletti, "Minimum mass design of tensegrity bridges with parametric architecture and multiscale complexity," pp. 124-132, ( ) 2014, with permission from Elsevier.]

ules). The paper first demonstrates the optimization algorithm and then gives numerical confirmations and examples. In particular, Latteur et al. proposed, as an illustration of their design methodology, the geometry of a tensegrity footbridge composed of a succession of elementary tensegrity simplex modules, called the snake footbridge (Fig. 4). The sinusoidal design of the deck comes naturally from the fact that it significantly increases not only the architectural value but also the headroom for pedestrians, since the upper nodes of the structures are not aligned.

This paper focuses mainly on topics related to tensegrity footbridges. Concerning morphological indicators and other design methodologies for tensegrity structures, a detailed state of the art can be found in Latteur et al. (2017).

The aim of this paper is to develop and use the design methodology demonstrated in Latteur et al. (2017) in order to prove the feasibility of footbridges composed of a succession of elementary tensegrity modules of the types simplex, quadruplex, hexaplex, and pentaplex (Figs. 4-7), with a suspended deck situated inside the main Class 2 tensegrity structures. In other words, the study focuses on selection of the types of tensegrity modules, their number $S$, and the ratios $L / H$ (span over height) that lead to the lightest and/or stiffest structures. The relevance of this type of construc- tion with respect to other types of structures, such as trusses, is discussed, and a practical example of a 40-m-long footbridge is given.

\section{Assumptions and Definitions}

\section{General Assumptions}

This paper considers any structure

- of span $L$, height $H$, and width $D$, considered after application of the prestress (Fig. 6) and composed of a number $S$ of elementary tensegrity modules of type simplex, quadruplex, pentaplex, or hexaplex (Fig. 5);

- with $n_{c}$ cables made of the same material of specific weight $\rho_{c}$, Young's modulus $E_{c}$, and design strength $\sigma_{c}$;

- with $n_{s}$ struts made of the same material of specific weight $\rho_{s}$, Young's modulus $E_{s}$, and design strength $\sigma_{s}$, and such that factor $u$ is defined as $u=\sigma_{c} / \sigma_{s}$;

- with materials having an elastic linear behavior, $\sigma_{c}$ and $\sigma_{s}$ coming, respectively, from the division of the characteristic yield strength by the adequate security coefficient (discussed in the next section); 

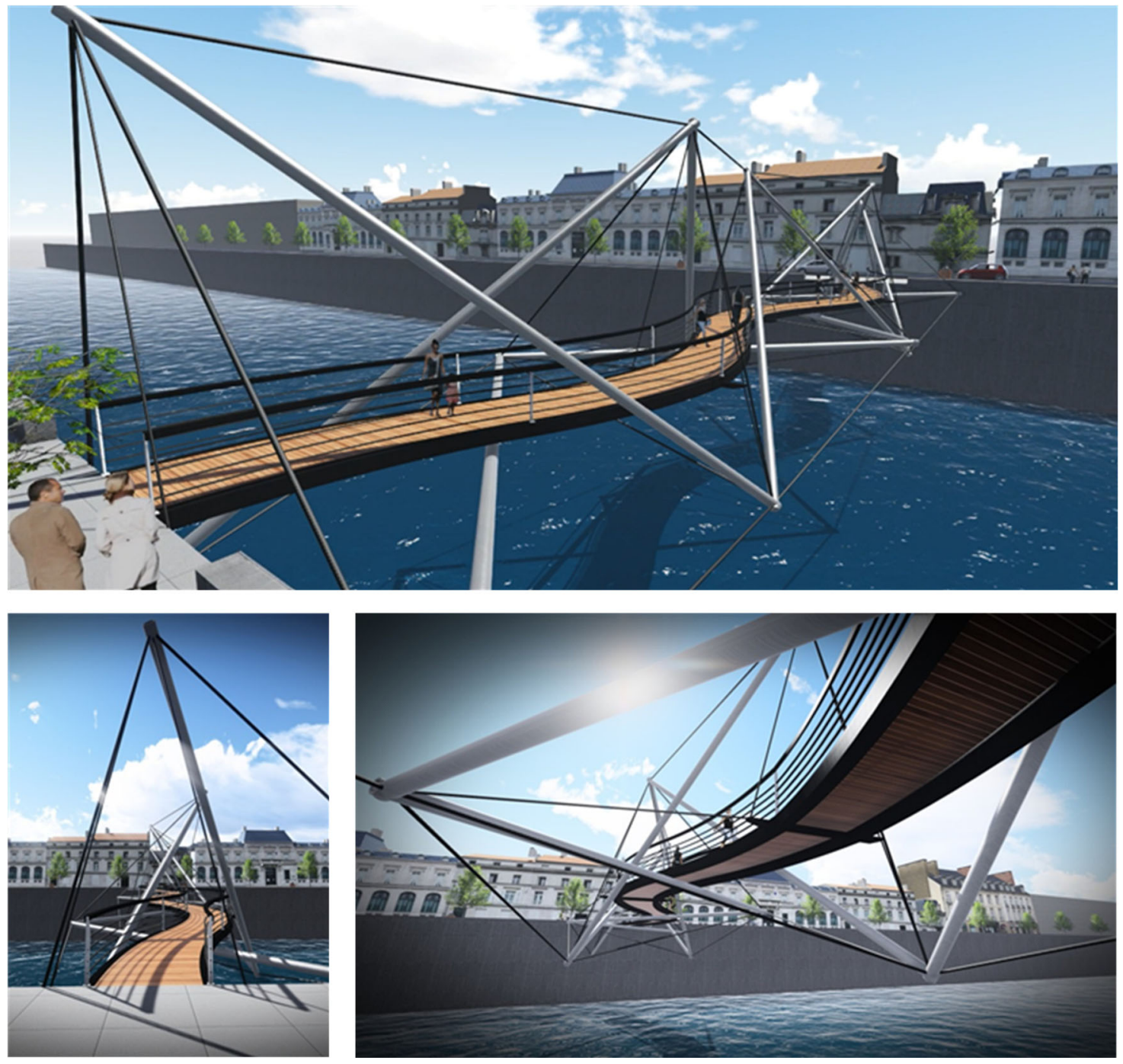

Fig. 4. Snake footbridge design proposed by Latteur et al. (2017).

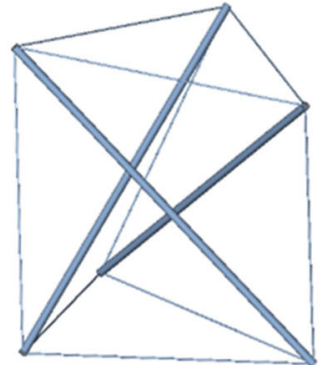

(a)

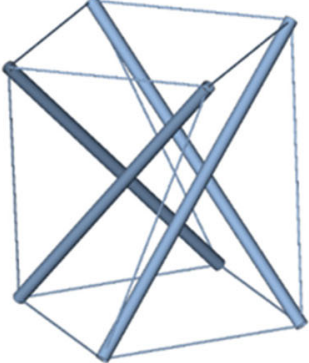

(b)

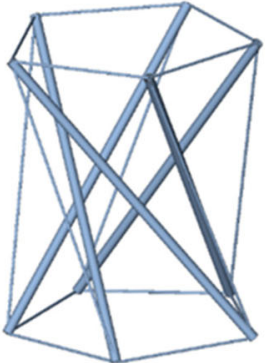

(c)

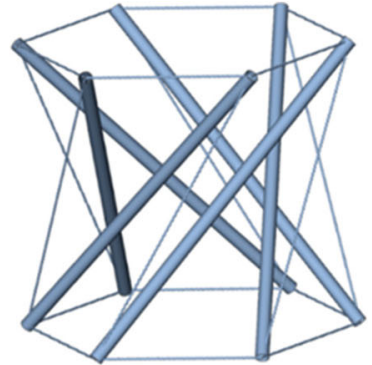

(d)

Fig. 5. Elementary tensegrity modules: (a) simplex; (b) quadruplex; (c) pentaplex; and (d) hexaplex.

- in which each cable (or any element remaining always in tension) of index $i$, subjected to a tension force $N_{c, i}$, has a crosssectional area $A_{c, i}$ related to the design criterion

$$
N_{c, i} / A_{c, i}=\sigma_{c}
$$

- in which each strut of index $i$ has a cross sectional area $A_{s, i}$ and a moment of inertia $I_{s, i}$, such that the form factor $q$, defined as
$q=I_{s, i} / A_{s, i}^{2}$ (Fig. 8), is supposed to be equal for all struts. The area $A_{s, i}$ of the strut of length $L_{0, i}$ subjected to a compression force $N_{s, i}$ is related to the design criterion (approximation of corrected Euler's critical load)

$$
\frac{N_{s, i}}{A_{s, i}}=\frac{1}{1+\Lambda_{i}^{2}} \sigma_{s} \quad \text { with }\left\{\begin{array}{l}
\Lambda_{i}=\lambda_{i} /\left(\pi \sqrt{E_{s} / \sigma_{s}}\right) \\
\lambda_{i}=L_{0, i} \sqrt{A_{s, i} / I_{s, i}}
\end{array}\right.
$$



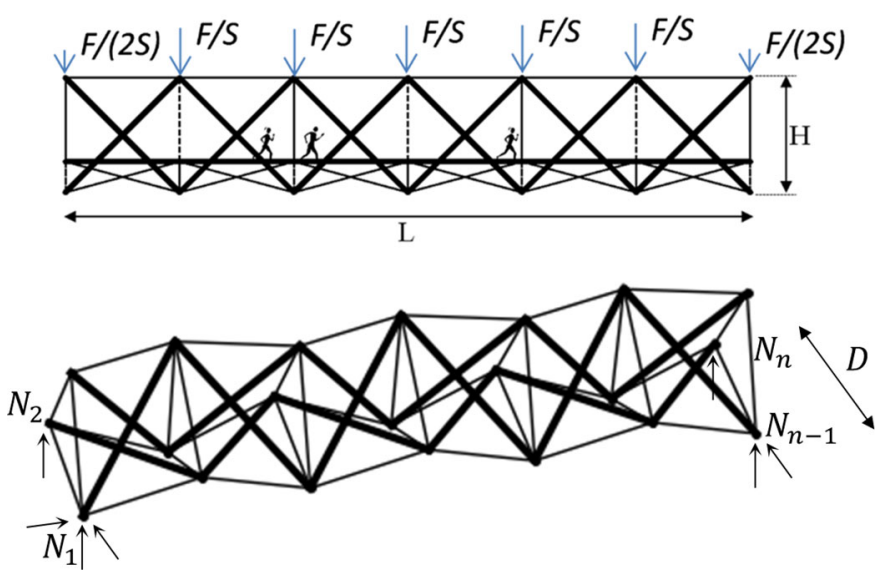

Fig. 6. Footbridge composed of a number $S=6$ of elementary tensegrity simplex modules and subjected to a total external load $F$ distributed on the upper nodes. The distribution of $F$ depends on the topology (see details in Fig. 7).

- in which cables and struts are designed according to Eqs. (1) and (2) and, although the methodology allows a fully stressed design, in this paper

all struts have the same cross-sectional area $A_{s}$ (and diameter $d_{s}$ ), provided by the most stressed strut, and

- longitudinal cables have the same cross-sectional area $A_{c, l}$ (and diameter $d_{c, l}$ ) and transversal cables have the same crosssectional area $A_{c, t}$ (and diameter $d_{c, t}$ ) (this assumption comes from the observation that the longitudinal cables are usually more stressed than the transversal ones);

- with a maximum deflection $\delta$ somewhere, for instance at midspan and vertically, considering that $\delta$ is induced by both external load $\tilde{\mathbf{F}}$ and self-weight $\tilde{\mathbf{G}}$;

- related to an indicator of buckling $\Psi$ developed by Latteur (2000), and such that $\Psi=\sigma_{s} L / \sqrt{q E_{s} F}$; and

- with a total volume $V$ of materials (cables and struts).

\section{External Loads, Self-Weight, Prestress, and Security Coefficients}

The usual structural design methodology consists of calculating the cross sections according to ultimate limit state (ULS) combinations and limiting the deflections according to serviceability limit state (SLS) combinations. Both depend on multiplication factors defined in the codes, which are not the same from one country to another and which have particular values for dead loads, live loads, wind, snow, and other load cases. The ratio between ULS and SLS combinations usually varies between 1.35 and 1.5. This way of designing must also take into account security regarding both accidental loss of prestress and accidental excessive prestress, as well as security coefficients on the characteristic yield strengths of materials (which usually vary from 1 to 1.5 , depending on the material). Globally, such ULS/SLS design is not easy to associate with an optimization methodology. Indeed, the assumptions about the multiplication factors related to ULS and SLS combinations, the range of security to consider for the prestress, and the security coefficients to consider for the materials pollute the analysis and the objective comparison between the different topologies. This is the reason why, in this paper, ULS and SLS combinations are not considered: live load $\tilde{\mathbf{F}}$ and self-weight $\tilde{\mathbf{G}}$ are not multiplied with any multiplication factor. However, a way to consider all those uncertainties is to include them in a single global security coefficient applied on the materials only. In particular, for the results given in this paper, steel with a char- acteristic yield strength of $355 \mathrm{MPa}$ is considered both for cables and struts (this assumption is discussed in section "Discussion and Conclusion"), associated with a global security coefficient of 1.5, which means

- a design strength equal to $\sigma=\sigma_{c}=\sigma_{s}=355 / 1.5=237 \mathrm{MPa}$;

- an elastic modulus equal to $E=E_{c}=E_{s}=210,000 \mathrm{MPa}$;

- $\operatorname{ratios} E / \sigma=E_{c} / \sigma_{c}=E_{s} / \sigma_{s}=886$; and

- a specific weight $\rho=\rho_{c}=\rho_{s}=77 \mathrm{kN} / \mathrm{m}^{3}$.

The combination of the external load cases acting in the three directions $(x, y, z)$ on the $n$ nodes of the structure is given by vector $\tilde{\mathbf{F}}$, such as

$$
\tilde{\mathbf{F}}=F \tilde{\mathbf{f}}=F\left(t_{1}^{F}, t_{2}^{F}, \ldots, t_{i}^{F}, \ldots, t_{3 n}^{F}\right)
$$

in which $\left\{\begin{array}{l}\tilde{\mathbf{f}}=\left(t_{1}^{F}, t_{2}^{F}, \ldots, t_{i}^{F}, \ldots, t_{3 n}^{F}\right) \\ \sum\left|t_{i}^{F}\right|=1\end{array}\right.$

Values of $F t_{i}^{F}$ are specified in Fig. 7 for each topology.

Self-weight is taken into account by assuming that each strut or cable connected to a node adds to this node a vertical load equal to half of its own self-weight. Self-weight is therefore considered a new external load case $\tilde{\mathbf{G}}$ added to $\tilde{\mathbf{F}}, \tilde{\mathbf{G}}$ being a $3 n$-dimensional vector in which only the components related to the vertical axis are non-null.

A judicious choice of prestress scenario $\tilde{\mathbf{P}}$ is necessary to ensure the stability of the structure, to prevent some cables from slack when the external load case $\tilde{\mathbf{F}}$ and self-weight $\tilde{\mathbf{G}}$ are considered, and, finally, to reach the desired stiffness.

Vector $\tilde{\mathbf{P}}$ is composed of the $n_{c}+n_{s}$ values of the axial force $P_{i}$ in each element, cable, or strut (with tension for cables and compression for struts). Those values are thus the internal axial forces that exist before the application of the external load $\tilde{\mathbf{F}}$ and selfweight $\tilde{\mathbf{G}}$. The prestress scenario $\tilde{\mathbf{P}}$ is defined as follows:

$$
\begin{gathered}
\tilde{\mathbf{P}}=\beta F \tilde{\mathbf{p}}=\beta F\left(t_{1}^{p}, t_{2}^{p}, \ldots, t_{i}^{p}, \ldots, t_{n_{c}+n_{s}}^{p}\right) \\
\text { in which }\left\{\begin{array}{c}
\tilde{\mathbf{p}}=\left(t_{1}^{p}, t_{2}^{p}, \ldots, t_{i}^{p}, \ldots, t_{n_{c}+n_{s}}^{p}\right) \\
\quad \text { is the prestress state } \\
-1 \leq t_{i}^{p} \leq 1 \\
\beta \geq 0 \text { is the prestress level }
\end{array}\right.
\end{gathered}
$$

A self-stress mode is a particular value of $\tilde{\mathbf{p}}$ that maintains the initial geometry of the structure, while a self-stress state is either a selfstress mode or a combination of several self-stress modes.

For a prestress state $\tilde{\mathbf{p}}$ judiciously chosen, $\beta_{\min }$ is defined as the particular value of $\beta$ that leads to a situation in which the axial force into the least-tensioned cable after consideration of the external load $\tilde{\mathbf{F}}$ and self-weight $\tilde{\mathbf{G}}$ is equal to zero, which means that no cable slacks when $\beta=\theta \beta_{\min }$ and $\theta \geq 1$

In this paper, $\theta=1$ and $\beta=\beta_{\text {min }}$ will be assumed, and the way to find the value $\beta_{\min }$ is discussed in Latteur et al. (2017). The impact of $\theta \neq 1$ is discussed in the conclusion of this paper.

As detailed in Latteur et al. (2017), a way to numerically create a prestress scenario $\tilde{\mathbf{P}}$ is to numerically apply to some element $i$ of the structure an external axial force $F_{\mathrm{pre}, i}$ at its extremities.

The $\left(n_{c}+n_{s}\right)$ values of the external prestress force $F_{\text {pre }, i}$ can be defined by vector $\tilde{\mathbf{F}}_{\text {pre }}$ 
S1:
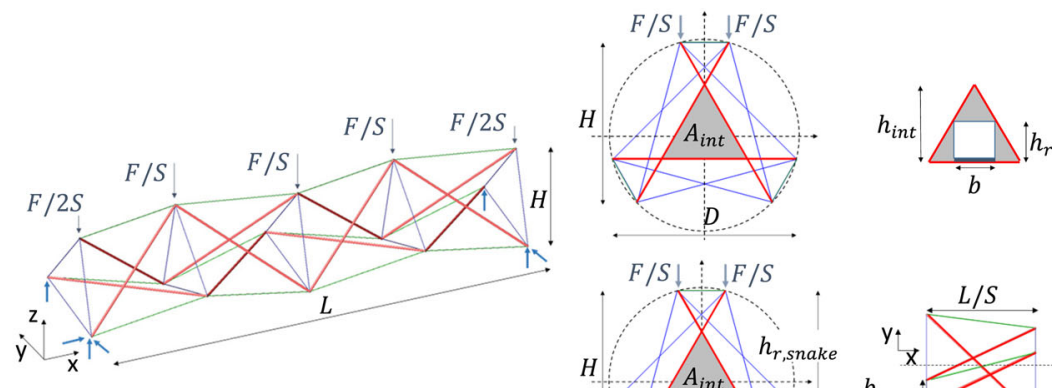

$h_{r}=0.46 H-0.87 b$

S2
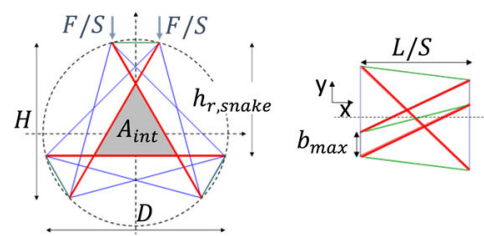

$h_{r, \text { snake }}=0.65 \mathrm{H}$

$b_{\max }=0.27 \mathrm{H}$

(a)
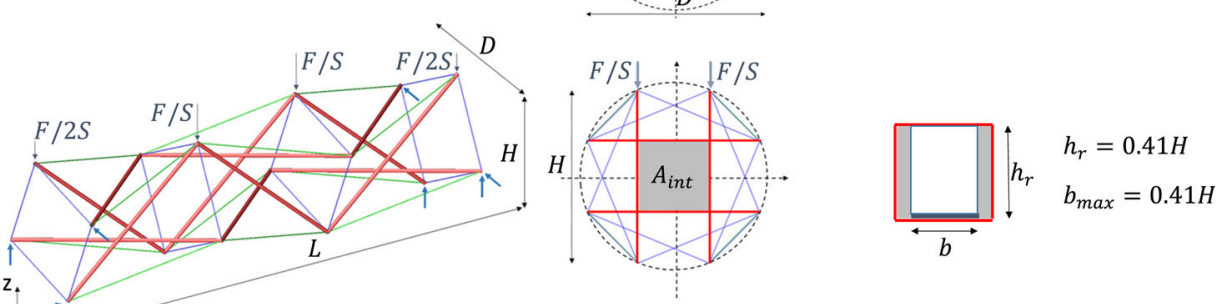

(b)

$$
\vec{y} \underset{\vec{x}}{2} \text {. }
$$
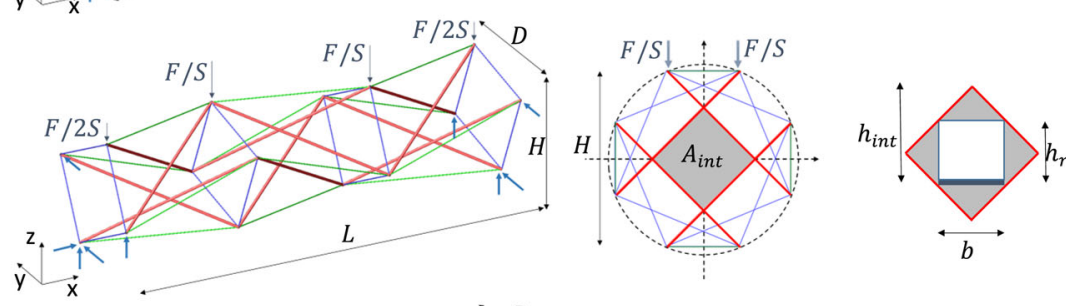

$h_{r}=0.59 H-b$

$b_{\max }=0.59 H \quad\left(\right.$ for $\left.h_{r}=0\right)$

$\left(h_{\text {int }}=0.59 H-0.5 b\right)$

(c)
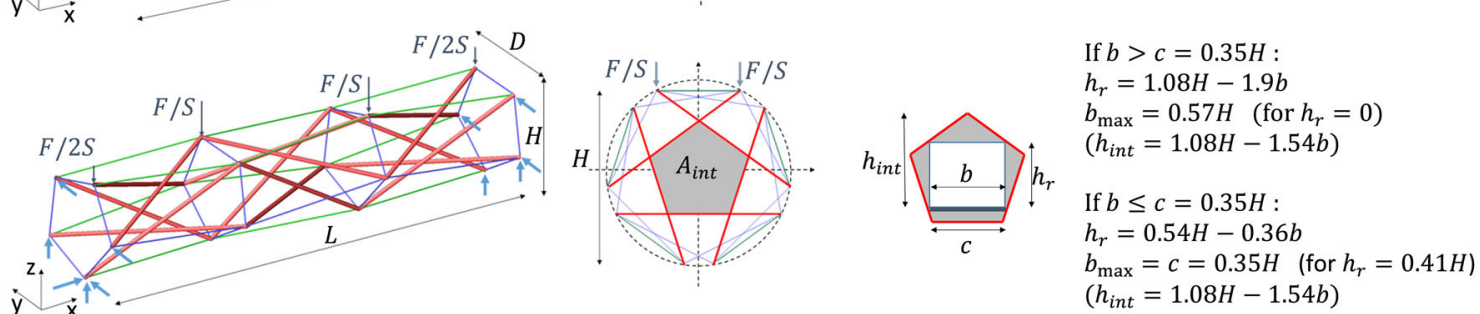

(d)

P1:
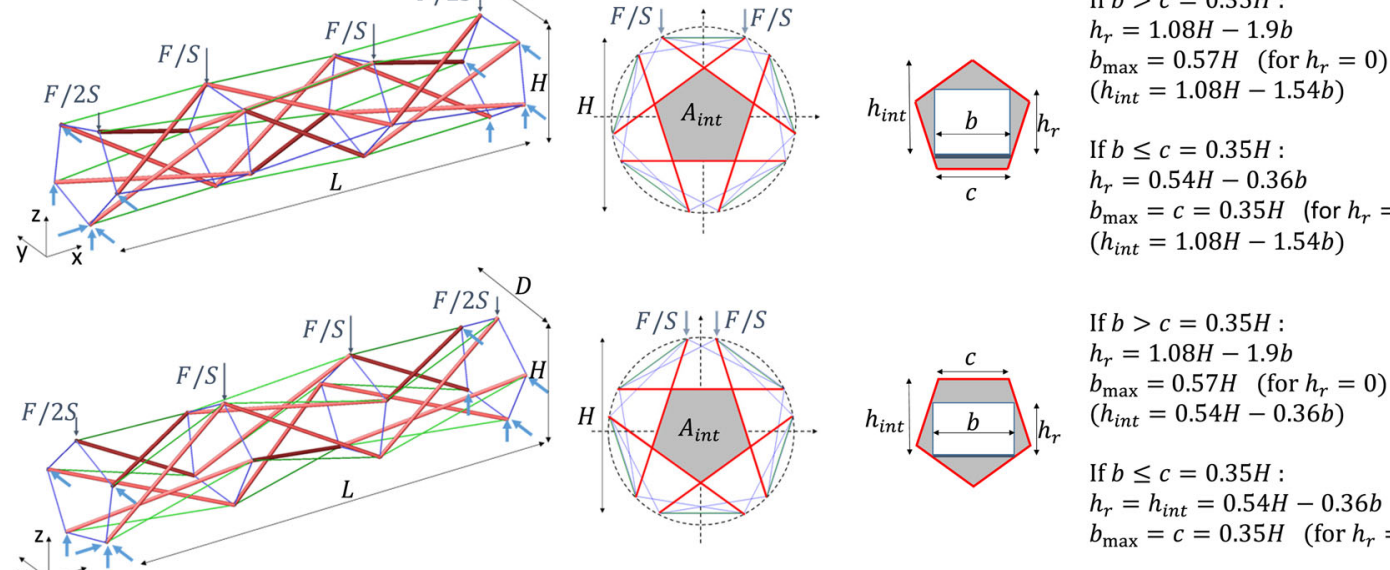

P2:
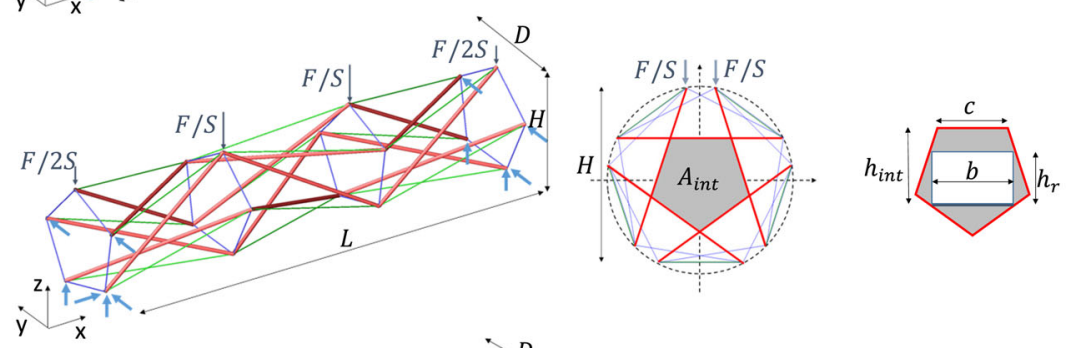

If $b \leq c=0.35 \mathrm{H}$

$h_{r}=h_{\text {int }}=0.54 \mathrm{H}-0.36 \mathrm{~b}$

$b_{\max }=c=0.35 H \quad\left(\right.$ for $\left.h_{r}=0.41 H\right)$

(e)
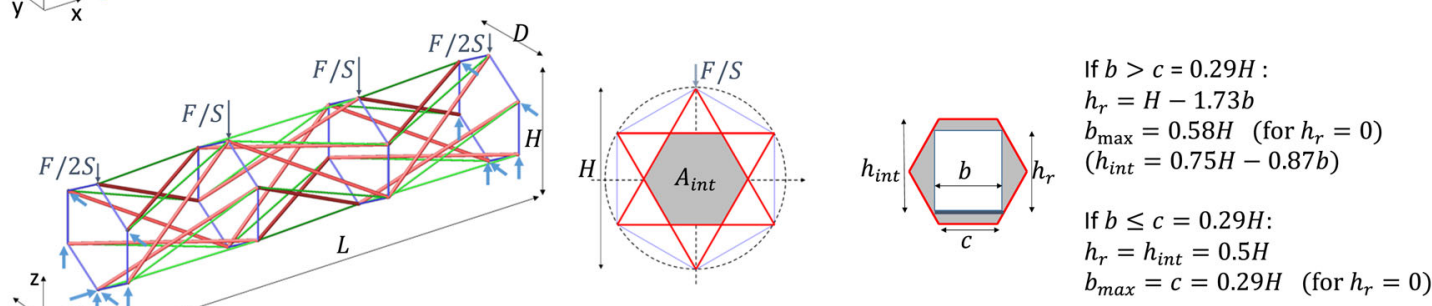

(f)

H1:

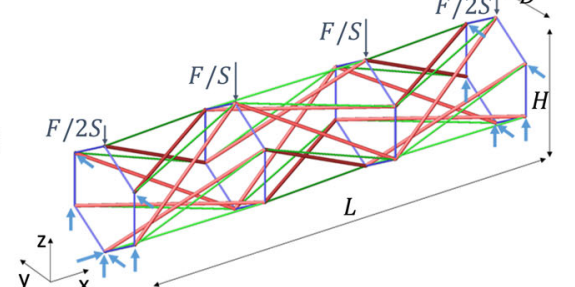

$b_{\max }=c=0.29 H \quad\left(\right.$ for $\left.h_{r}=0\right)$

$\mathrm{H} 2$ :
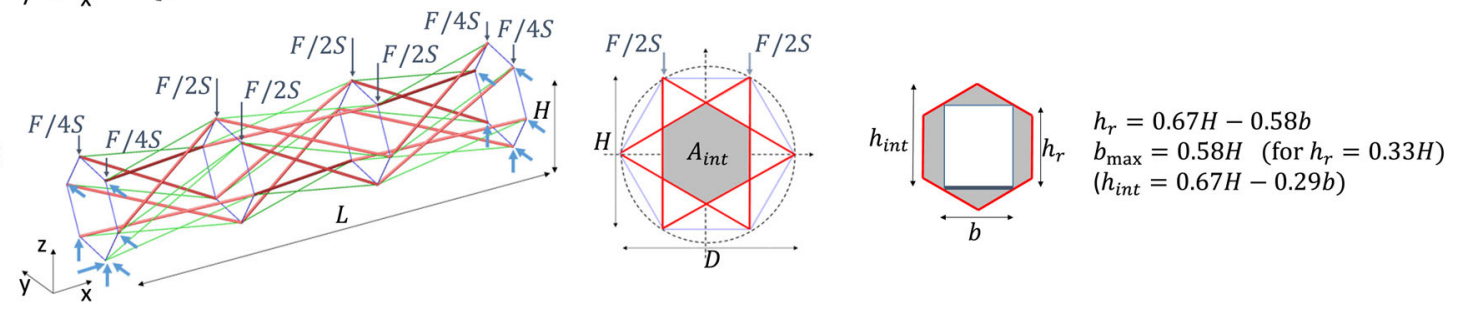

(g)

Fig. 7. Seven topologies of footbridges considered in this study and detailed in section "Assumptions and Definitions": (a) simplex S1 and S2 (S2 is similar to S1 but with a sinusoidal deck); (b) quadruplex Q1; (c) quadruplex Q2; (d) pentaplex P1; (e) pentaplex P2; (f) hexaplex H1; and (g) hexaplex H2. 


$$
\begin{gathered}
\tilde{\mathbf{F}}_{\text {pre }}=\beta_{\mathrm{pre}} F \tilde{\mathbf{f}}_{\mathrm{pre}}=\beta_{\mathrm{pre}} F\left(t_{1}^{\mathrm{pre}}, t_{2}^{\mathrm{pre}}, \ldots, t_{i}^{\mathrm{pre}}, \ldots, t_{n_{c}+n_{s}}^{\mathrm{pre}}\right) \\
\text { with }\left\{\begin{array}{l}
\tilde{\mathbf{f}}_{\mathrm{pre}}=\left(t_{1}^{\mathrm{pre}}, t_{2}^{\mathrm{pre}}, \ldots, t_{i}^{\mathrm{pre}}, \ldots, t_{n_{c}+n_{s}}^{\mathrm{pre}}\right) \\
-1 \leq t_{i}^{\mathrm{pre}} \leq 1 \\
\beta_{\mathrm{pre}} \geq 0
\end{array}\right.
\end{gathered}
$$

The external axial prestress forces $\tilde{\mathbf{F}}_{\text {pre }}$ are thus generating the in-

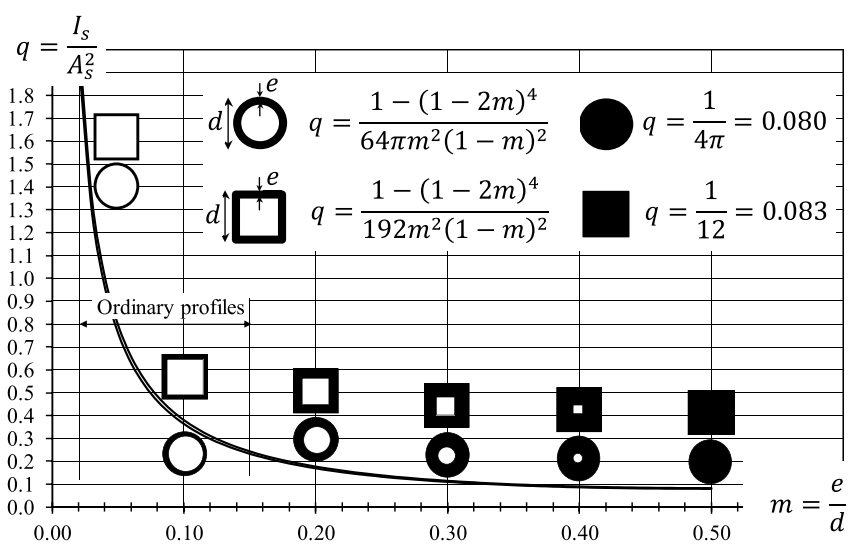

Fig. 8. Values of form factor $q=I_{s} / A_{s}^{2}$ for usual cross sections. ternal prestress forces $\tilde{\mathbf{P}}$ which may be different from $\tilde{\mathbf{F}}_{\text {pre }}$. The value $\beta_{\text {pre,min, }}$, just like $\beta_{\text {min }}$, is defined as the particular value of $\beta_{\text {pre }}$ that leads to a situation in which the axial force in the leasttensioned cable after application of the external load $\tilde{\mathbf{F}}$ and selfweight $\tilde{\mathbf{G}}$ is equal to zero, which means that no cable slacks.

The proposed design methodology considers that the choice of $\tilde{\mathbf{f}}_{\text {pre }}$ is arbitrary, chosen once and for all. Note that there may exist a better choice of $\tilde{\mathbf{f}}_{\text {pre }}$, which leads to a stiffer or lighter structure. In this paper, one assumes that the values of $\tilde{\mathbf{f}}_{\text {pre }}$ are null for the cables and identical for the struts: $\tilde{\mathbf{f}}_{\text {pre }}=(0, \ldots, 0,-1, \ldots,-1)$. This hypothesis corresponds to a practical situation in which only struts are equipped with a mechanical device that can elongate them.

\section{Supports, Topologies, Load Cases, and Headroom}

If $R_{s}$ is the number of reactions at supports, the degree of redundancy $D_{r}$ of such structures composed of $n$ nodes is given by the following equation:

$$
D_{r}=\left(n_{c}+n_{s}\right)+R_{s}-3 n
$$

For each topology, many different positions of the supports can be chosen. For instance, the support on node $N_{2}$ is not essential to ensure the global stability of the structure shown in Fig. 6. However, support on $N_{2}$ greatly improves the structural behavior in terms of stiffness [in this case, $D_{r}=(39+18)+7-3 \times 21=1$ ].

In order to perform a relevant comparison of the structures made

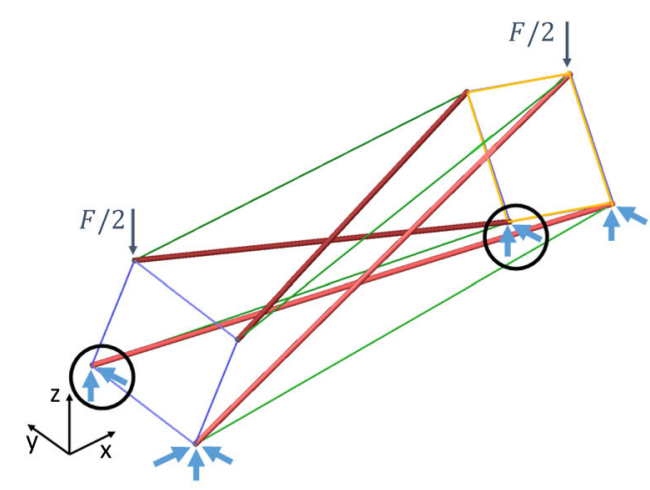

(a)

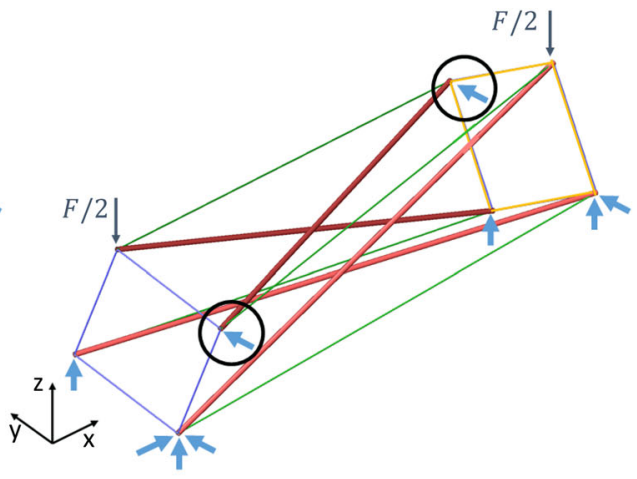

(b)

Fig. 9. For a same degree of redundancy, the position of the supports (shown by arrows) strongly influences the structural behavior. From this point of view, (b) is much better than (a).

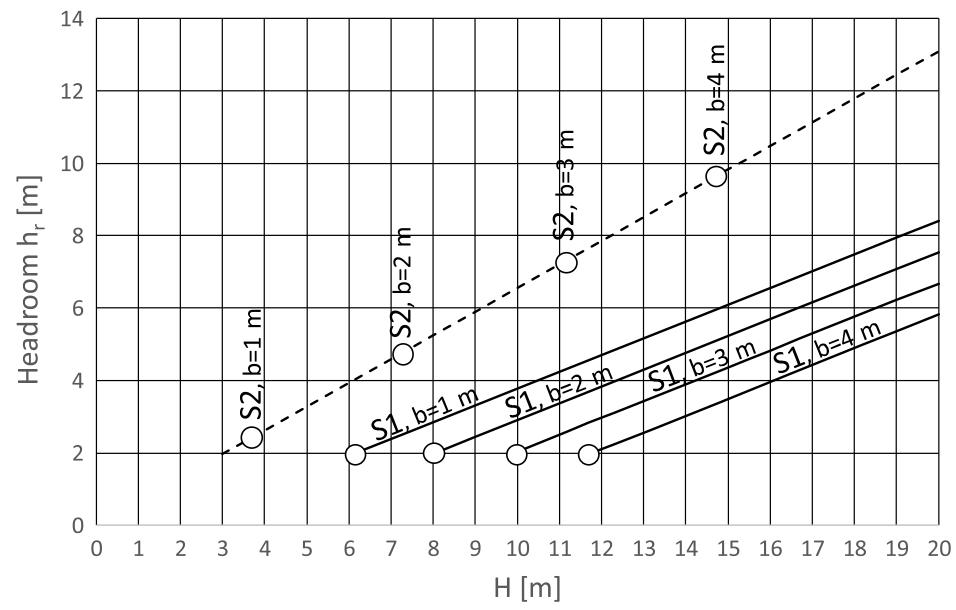

Fig. 10. Headroom inside a simplex for straight deck configuration (S1) and snake deck configuration (S2). 
of different topologies (for example, simplex and quadruplex), a same degree of redundancy $D_{r}$ equal to 1 is arbitrarily chosen in this paper.

Again, for a same degree of redundancy, several positions of the supports can be chosen, but some of them are not adequate. Fig. 9 shows the simplified situation of a unique quadruplex in which both situations correspond to $D_{r}=1$ and in which the prestress is introduced via an identical elongation of the four struts. The structure of Fig. 9(a) has very large displacements of the upper nodes when the external load $\tilde{\mathbf{F}}$ is applied, while the structure of Fig. 9(b) has much more limited displacements. This example shows that the best choice of the position of the supports remains difficult to formalize.

This paper studies and compares seven different topologies associated with their particular support positions and load distributions, as shown in Fig. 7. Concerning the distribution of the external load $\tilde{\mathbf{F}}$, it is assumed that each external force acts vertically on the upper nodes. Practically, this could be different, since the deck can be suspended from the upper nodes with more than one cable, giving to the resultant force a horizontal component. The deck could also be supported by the lower and/or lateral nodes, which is not considered in this study.

An important topic is that the structures that do not allow a sufficient deck width $b$ and headroom $h_{r}$ should be omitted from the results of the optimization process. Fig. 7 gives the formulas to evaluate the headroom for each topology. In this paper, a minimum width $b=2 \mathrm{~m}$ and minimum headroom $h_{r}=2 \mathrm{~m}$ will be considered in the sections "General Comparison of the Seven Topologies" and "Practical Example: 40-m-Span Tensegrity Footbridge." The value $h_{r}=2 \mathrm{~m}$ is indeed the absolute minimum value, below which the construction of a footbridge would be irrelevant (in many houses, door openings have a 2-m height).

A solution to increase the headroom for topology S1 is to change the straight geometry of the deck into a sinusoidal one (S1 then becomes the S2 topology), as shown in Fig. 4.

Fig. 10 shows that

- for S1 and S2, sufficient headroom (>2 m) and deck width $(>2 \mathrm{~m})$ need a height $H$ of the structure above $8 \mathrm{~m}$ and $7 \mathrm{~m}$, respectively (note, however, that other topologies, such as Q1, may offer better results in terms of $h_{r}$ and $b$ and may allow the reduction of $H$ to values around $5 \mathrm{~m}$, although these topologies are not efficient, as will be shown); and

- considering such structures for spans shorter than $20 \mathrm{~m}$ makes no sense, since the deck width and/or the headroom would be insufficient. Indeed, optimum structures are found for slenderness $\mathrm{L} / \mathrm{H}$ that range between 3 and 7 , as shown in the results of this article.

\section{Dynamic Behavior}

Footbridges are sensitive to pedestrian loading, and the accelerations and deformation amplitudes that they experience must be limited. Although vibration criteria are implicitly covered in SLSs, some codes suggest, especially in the predesign stage, to keep the natural frequencies of the footbridge outside a critical frequency band. For instance, Eurocode 0 (CEN 1990) suggests avoiding the ranges 0$2.5 \mathrm{~Hz}$ and $0-5 \mathrm{~Hz}$, respectively, for horizontal and vertical vibrations. Other design guidelines, such as the Sétra (Sétra/AFGC 2006), HIVOSS (Heinemeyer et al. 2009), and Tricon (Van den Broeck and De Roeck 2012), give more precise information and are, therefore, usually preferred. For instance, the Sétra guideline recommends natural frequencies associated with horizontal vibrations outside the range $0.5-1.1 \mathrm{~Hz}$ and those associated with vertical vibrations outside the range $1.7-2.1 \mathrm{~Hz}$, as well as its first few integer multiples. At the end of this paper (see the section "Practical Example: 40-m-Span Tensegrity Footbridge"), a modal analysis of an S2 tensegrity footbridge is reported and indicates that it features natural frequencies outside the critical ranges. For this reason, the dynamic behavior criterion is not included in the discussion of optimality of this paper.

\section{Allowable Deflections}

Limitation of the deflection is necessary for several reasons: behavior of supports, evacuation of water, aesthetic of the structure, vibrations, and comfort of the pedestrians.

Concerning the live loads only, the deflection is often limited to values between $L / 250$ and $L / 750$, depending on the standards, or even from one project to another. For instance, Section 5 of AASHTO (2009) recommends that "The deflection of the bridge due to the unfactored pedestrian live loading shall not exceed 1/360 of the span length."

Concerning the deflection $\delta$ due to dead loads combined with live loads, it is linked to the fundamental frequency $v$ of a footbridge and can be approximated with the equation $2 \pi v \cong \sqrt{9.81 / \delta}$ [where $v$ is expressed in hertz; 9.81 is in meters per second squared $\left(\mathrm{m} / \mathrm{s}^{2}\right)$; and $\delta$ is in meters]. This equation shows that, for a minimum fundamental frequency equal to $2.5 \mathrm{~Hz}, \delta$ should be limited to $0.04 \mathrm{~m}$, which means $L / 500$, for a span of $20 \mathrm{~m}$. If the span or the required minimum fundamental frequency is higher, the stiffness criteria should be even stricter. Consequently, for large spans, the limitation of the deflection may be too restrictive to guarantee a minimum comfort for the pedestrians, and the vibration problem must then be solved by use of viscous or tuned mass dampers. Whatever the span, it is thus largely accepted that the stiffer the structure is, the better it is.

In this paper, a footbridge with a deflection under both live loads and self-weight greater than $L / 500$ is considered inefficient. This is of course an arbitrary choice, but globally it is in accordance with the foregoing comments.

However, this study shows that the best structures in terms of self-weight (essentially the structures composed of simplex modules) have surprisingly good stiffness, generally with a deflection lower than $L / 500$. The removal of the few cases with $\delta / L>1 / 500$ in the section "General Comparison of the Seven Topologies" does not change the global results and conclusions of the paper.

\section{Design Methodology Using Morphological Indicators}

It has been proven (Latteur et al. 2017) that, for a given structure composed of $S$ elementary tensegrity modules of span $L$, height $H$, and width $D$, composed of materials of mechanical characteristics $\left(E_{c}, \sigma_{c}, E_{s}\right.$, and $\left.\sigma_{s}\right)$, subjected to an external load $\tilde{\mathbf{F}}=F \tilde{\mathbf{f}}$ and a prestress scenario $\tilde{\mathbf{P}}=\beta F \tilde{\mathbf{p}}$, and with struts and cables designed according to Eqs. (1) and (2),

- the deflection at midspan (or anywhere else) $\delta / L$ depends only on seven dimensionless numbers, according to

$$
\frac{\delta}{L}=f\left(\frac{L}{H}, \frac{L}{D}, \Psi, \frac{E_{c}}{\sigma_{c}}, \frac{E_{s}}{\sigma_{s}}, S, \beta \tilde{\mathbf{p}}\right)
$$

- the volume $V$ of materials, defined by its indicator of volume $W$, depends only on the same seven dimensionless numbers and on $u=\sigma_{c} / \sigma_{s}$, according to

$$
W=\frac{\sigma_{s} V}{F L}=f\left(\frac{L}{H}, \frac{L}{D}, \Psi, \frac{E_{c}}{\sigma_{c}}, \frac{E_{s}}{\sigma_{s}}, S, \beta \tilde{\mathbf{p}}, u\right)
$$

where $\Psi$ is the indicator of buckling (Latteur et al. 2000, 2001) such that $\Psi=\sigma_{s} L / \sqrt{q E_{s} F}$.

This methodology allows us to easily find the stiffest or the lightest structure thanks to the efficiency curves shown in Fig. 11. Indeed, assuming that 


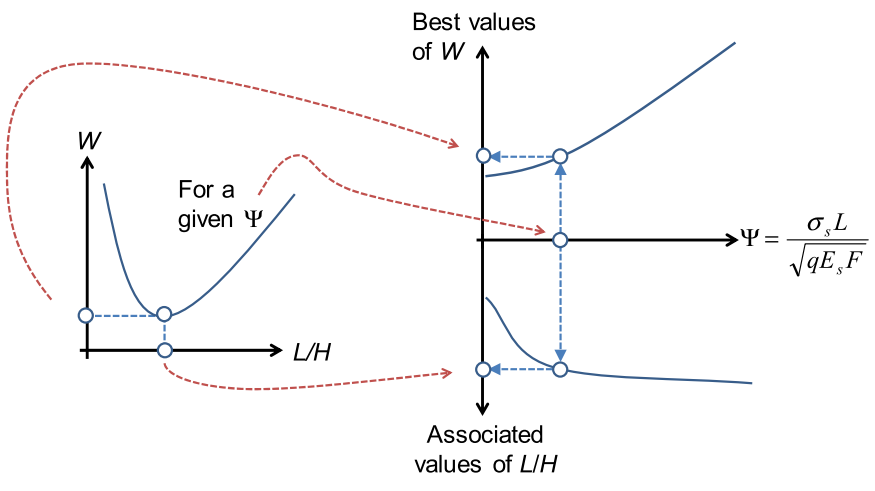

Fig. 11. For a given practical case related to a given value of $\Psi$, the efficiency curve gives the best value $L / H$ and the associated (best) value of the indicator of volume $\mathrm{W}$.
- the span $L$ and the load case $\tilde{\mathbf{F}}$ of a given project are known,

- the materials are chosen $\left(E_{c} / \sigma_{c}, E_{s} / \sigma_{s}, u\right)$,

- the prestress level and state, respectively, $\beta$ and $\tilde{\mathbf{p}}$ are arbitrarily fixed, as explained in the assumptions, and

- $D$ is proportional to $H$ for a given topology of structures,

Eqs. (3) and (4) become, for a given number $S$ of elementary modules

$$
\frac{\delta}{L}=f\left(\frac{L}{H}, \Psi\right)
$$

and

$$
W=\frac{\sigma_{s} V}{F L}=f\left(\frac{L}{H}, \Psi\right)
$$

Using Eqs. (5) and (6), the optimization and the design process are thus greatly simplified, since the relative deflection $\delta / L$ and the indicator of volume $W$ depend only on the two parameters $L / H$ and $\Psi$. This is illustrated in Fig. 11 for the indicator of volume $W$.

The goal of this paper is to compare the structural performances

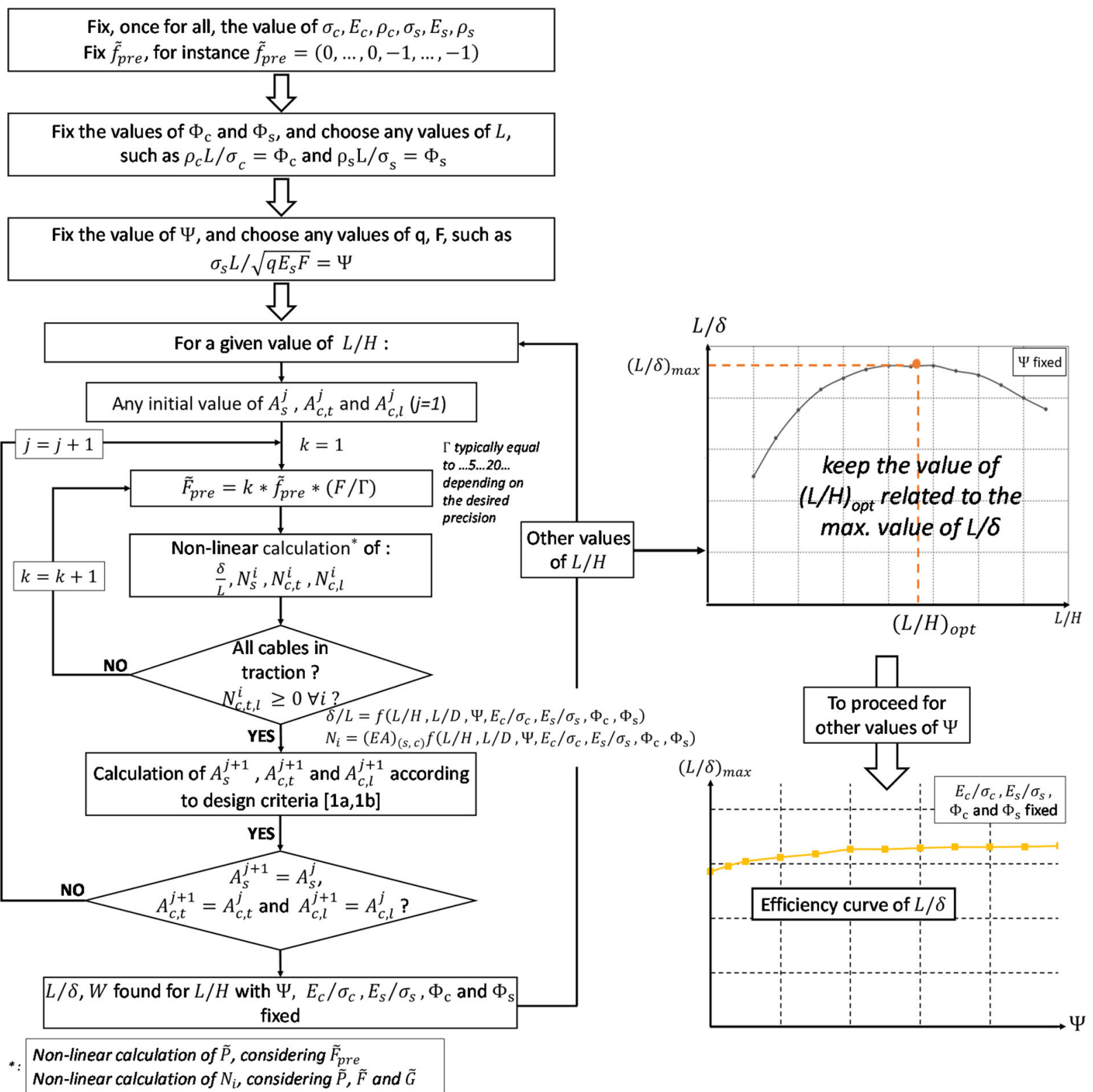

Fig. 12. Numerical algorithm used to find the value of $\left[L / H,(L / \delta)_{\max }\right]$ related to given values of $\Psi$ and $\Phi$. 

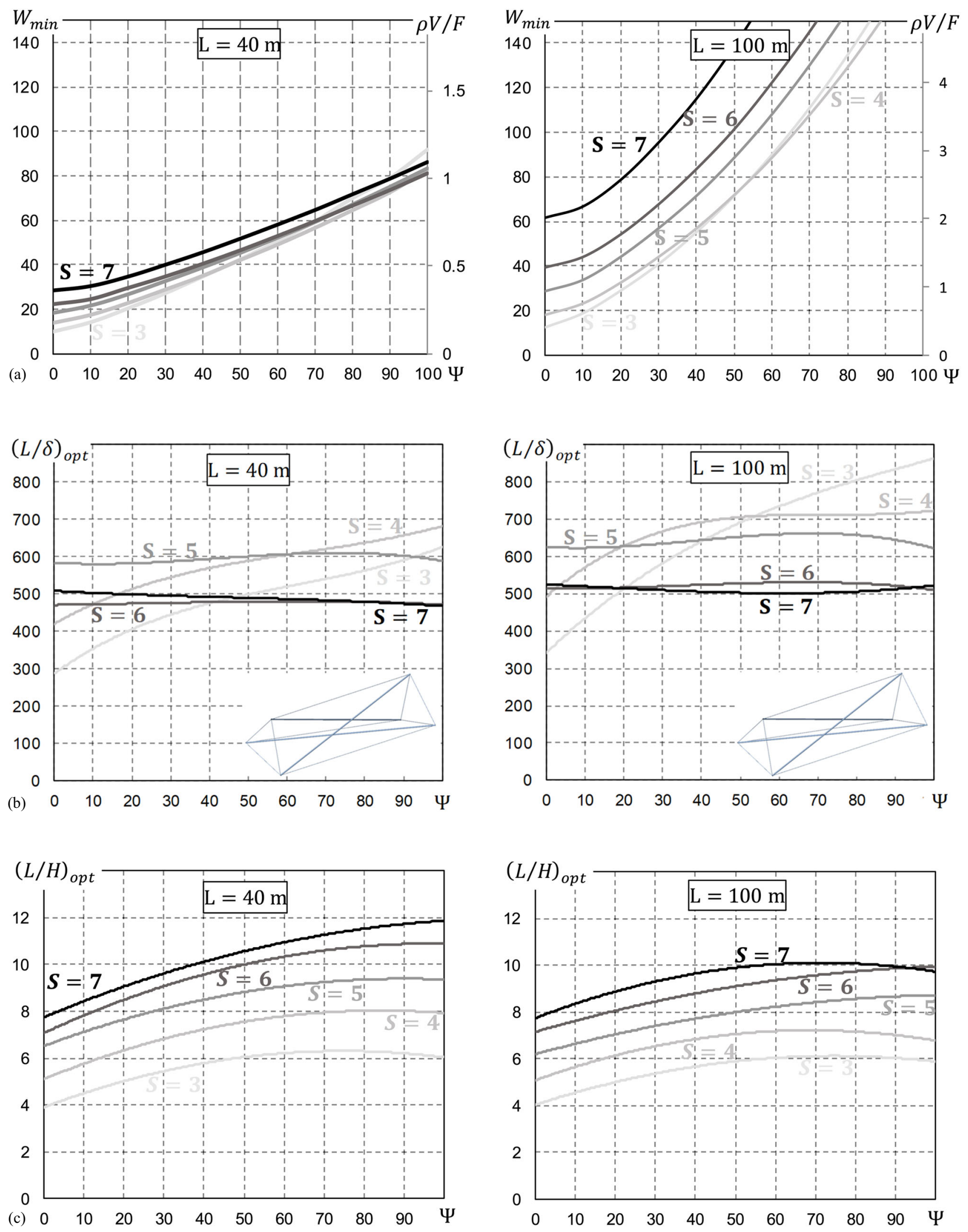

Fig. 13. Family $\mathrm{S} 1 / \mathrm{S} 2$, efficiency curves of $W$ for $L=40 \mathrm{~m}\left(\Phi=130 \times 10^{-4}\right)$ and $L=100 \mathrm{~m}\left(\Phi=325 \times 10^{-4}\right)$ : (a) efficiency curves of $W$ and $\rho V / F$; (b) associated values of $L / \delta$; and (c) associated values of $L / H$. 
(stiffness and volume) of the seven topologies of structures shown in Fig. 7 thanks to the efficiency curves and to discuss the relevance of such structures for footbridge applications.

The developments of Latteur et al. (2017) consider only the external load case $\tilde{\mathbf{F}}$ for the optimization process. This means a structure that is optimized only for the external load case $\tilde{\mathbf{F}}$ might no longer be optimum when the self-weight $\tilde{\mathbf{G}}$ is combined with $\tilde{\mathbf{F}}$, since self-weight $\tilde{\mathbf{G}}$ can reach a nonnegligible percentage of $\tilde{\mathbf{F}}$ for large spans. However, a solution can be found, as explained in the discussion that follows.

It is assumed that gravity is acting in the Z-direction and that the bending of the elements due to their self-weight can be neglected. For any structure, self-weight can thus be taken into account by assuming that each strut or cable connected to a node adds to this node a vertical load equal to half of its own self-weight. The process is of course iterative: given an external load $\tilde{\mathbf{F}}$, one can calculate the required total volume $V$ of materials and its associated self-weight equivalent load case $\tilde{\mathbf{G}}$, which leads to a new calculation of the structure subjected to $(\tilde{\mathbf{F}}+\tilde{\mathbf{G}})$, and so on until the results converge toward a final value of $\tilde{\mathbf{G}}$.

Taking self-weight into account keeps the design methodology described in Latteur et al. (2017) valid. Indeed,

- half of the self-weight of a strut or cable of index $i$ of volume $V_{i}$ is equal to $\rho V_{i} / 2$; and

- given the fact that the part $W_{i}$ of the indicator of volume related to an element $i$ of a structure subjected to $\tilde{\mathbf{F}}$ is equal to

$$
\begin{gathered}
W_{i}=\frac{\sigma_{s} V_{i}}{F L}=f\left(\frac{L}{H}, \frac{L}{D}, \Psi, \frac{E_{c}}{\sigma_{c}}, \frac{E_{s}}{\sigma_{s}}, S, \beta \tilde{\mathbf{p}}, u\right), \text { one gets } \\
\frac{\rho V_{i}}{2}=\frac{\rho L}{\sigma} \times \frac{F}{2} \times f\left(\frac{L}{H}, \frac{L}{D}, \Psi, \frac{E_{c}}{\sigma_{c}}, \frac{E_{s}}{\sigma_{s}}, S, \beta \tilde{\mathbf{p}}, u\right)
\end{gathered}
$$

If $\Phi_{c}=\rho_{c} L / \sigma_{c}$ and $\Phi_{s}=\rho_{s} L / \sigma_{s}$ are defined, respectively, as the indicators of self-weight (Latteur 2000) of the cables and the struts, upper relations show that self-weight $\tilde{\mathbf{G}}$ coming from an external load $\tilde{\mathbf{F}}$ is acting as a new load case that is proportional to $F$ and depends only on parameters $L / H, L / D, \Psi, E_{c} / \sigma_{c}, E_{s} / \sigma_{s}, S, \beta \tilde{\mathbf{p}}, u$, $\Phi_{c}$, and $\Phi_{s}$. This allows us to extend Eqs. (3) and (4) as follows:

$$
\begin{gathered}
\frac{\delta}{L}=f\left(\frac{L}{H}, \frac{L}{D}, \Psi, \frac{E_{c}}{\sigma_{c}}, \frac{E_{s}}{\sigma_{s}}, S, \beta \tilde{\mathbf{p}}, u, \Phi_{c}, \Phi_{s}\right) \\
W=\frac{\sigma_{s} V}{F L}=f\left(\frac{L}{H}, \frac{L}{D}, \Psi, \frac{E_{c}}{\sigma_{c}}, \frac{E_{s}}{\sigma_{s}}, S, \beta \tilde{\mathbf{p}}, u, \Phi_{c}, \Phi_{s}\right)
\end{gathered}
$$

It is interesting to note that taking $\tilde{\mathbf{G}}$ into account makes this time $\delta / L$ dependent on factor $u$.

Note that if both cables and struts are considered in steel with $E=210 \mathrm{GPa}, \sigma=237 \mathrm{MPa}$, and $\rho=77 \mathrm{kN} / \mathrm{m}^{3}$, the indicator of self-weight is equal to $\Phi=0.325 \times 10^{-6} \times L$ (with $L$ in mm). For spans limited to $100 \mathrm{~m}$, the indicator of self-weight is thus limited to

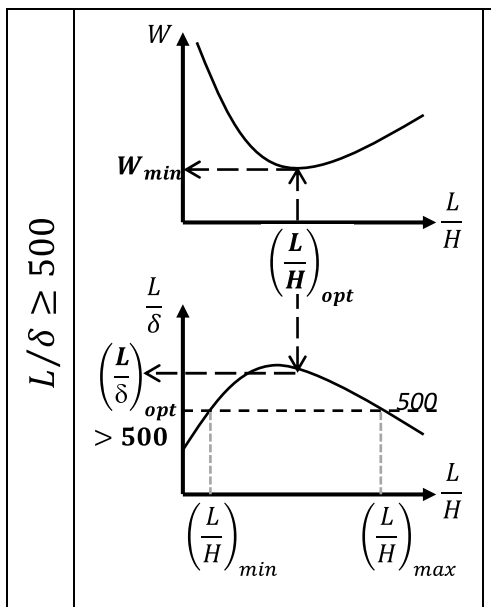

(a)

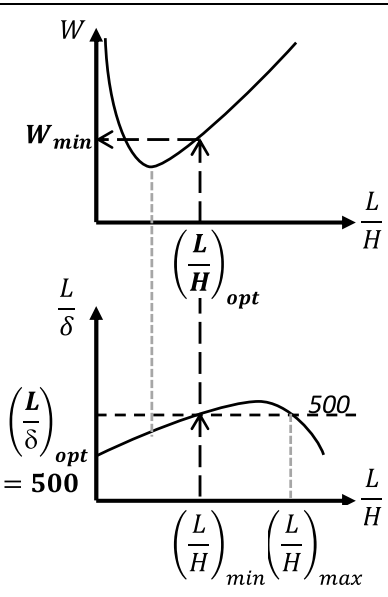

(b)

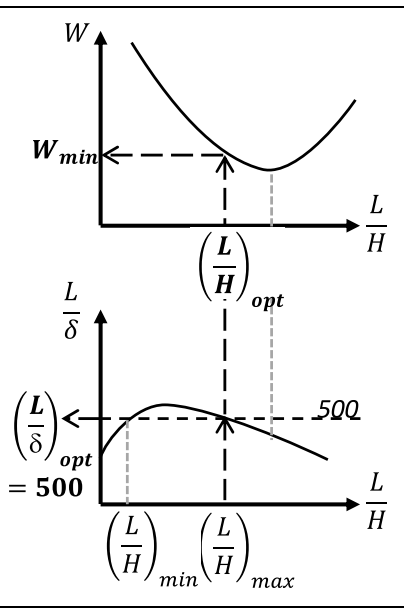

(c)

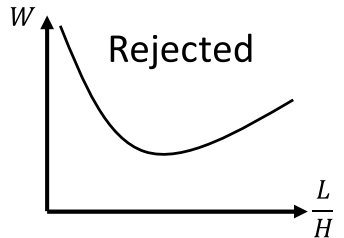

$L$

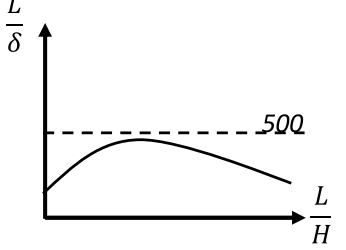

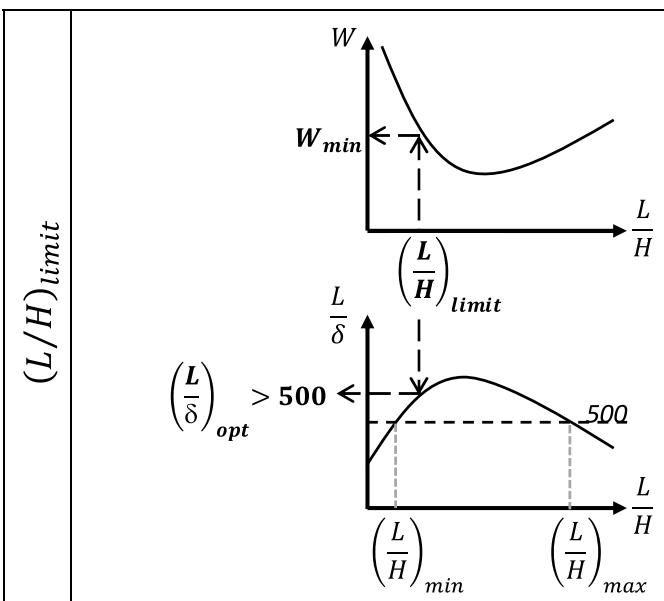

(e)

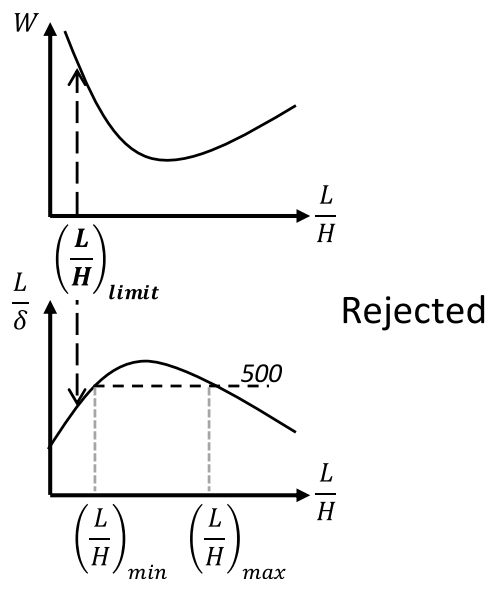

(d)

Fig. 14. Construction of efficiency curve of $W$ by selecting the best $L / H$ for a fixed $\Psi$ with the constraints $\delta \leq L / 500, h_{r} \geq 2 \mathrm{~m}$, and $b \geq 2 \mathrm{~m}$. 
$325 \times 10^{-4}$. Furthermore, previous study (Latteur et al. 2000) has shown that values of $\Psi$ higher than 100 are related to very heavy and low-efficient structures.

A very interesting consequence of the existence of the indicator of self-weight $\Phi$ is that it can be used to quantify the ratio between the total self-weight $\rho V$ of the structure and the total external load $F$

$$
\frac{\rho V}{F}=\rho \frac{V}{F}=\rho \frac{W L}{\sigma_{s}}=\Phi_{s} W=f\left(\frac{L}{H}, \frac{L}{D}, \Psi, \frac{E_{c}}{\sigma_{c}}, \frac{E_{s}}{\sigma_{s}}, S, \beta \tilde{\mathbf{p}}, u, \Phi_{c}, \Phi_{s}\right)
$$

\section{Numerical Algorithm}

Fig. 12 summarizes the algorithm used by the software that the authors developed, already partly presented in Latteur et al. (2017) but here modified to take into account self-weight as a load case added to the external load case. The code was developed in Python, and Fig. 12 shows the numerical optimization process that leads to the results presented further.

The algorithm of Fig. 12 can be summarized as follows (for a given number $S$ of elementary tensegrity modules):

- materials are chosen; in this paper, steel $\left(E / \sigma=E_{c} / \sigma_{c}=886\right.$ and $u=1$ ) is considered;

- the values of $\Psi, \Phi$, and $L / H$ are first chosen within the ranges $0 \leq \Psi \leq 100,0 \leq \Phi \leq 325 \times 10^{-4}$, and $0<L / H \leq 15$;

- the value of $\Phi$ automatically gives the value of $L(\Phi=0.325 \times$ $\left.10^{-6} \times L\right)$

- factors $q$ and $F$ are arbitrarily chosen in order to respect the value of $\Psi$

$$
\Psi=\sigma L / \sqrt{q E F} \rightarrow q F=(\sigma L / \Psi)^{2} / E
$$

- the value of $L / H$ gives the value of $H$;

- the iterative calculation loops give $\delta / L$ (of $L / \delta$ ) at midspan (and $W$ ) for those precise values of $\Psi, \Phi$, and $L / H$;

- if the process is repeated for each value of $L / H$, the couple (best $L / H$, best $\delta / L$ ) or (best $L / H$, best $W$ ) can be found for each value of $\Psi$ and reported on an efficiency curve. One efficiency curve is related to a single value of $\Phi$ (or the span $L$ ) and a single value of $E / \sigma ;$ and

- the same algorithm can be used for several values of $S$, for instance, 2, 3, 4, 5, and 6 .

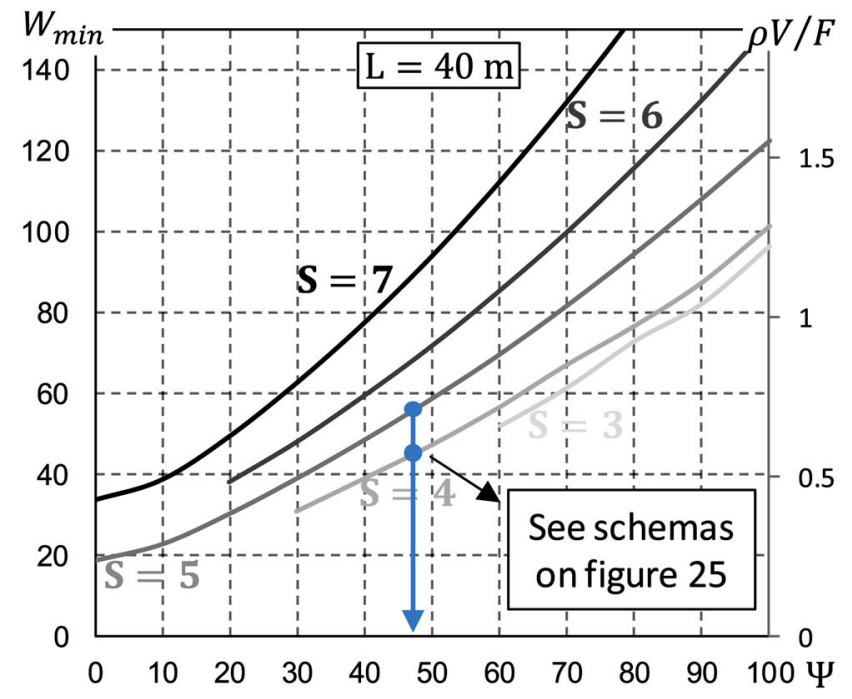

\section{Results for Family S1/S2 (Simplex)}

The possible information resulting from analysis of the computations done for each of the seven topologies (Fig. 7) is very dense: for example, the variations of $\delta / L$ and $W$ in function of $L / H$ for a fixed $\Psi$, the efficiency curves of $\delta / L$ associated with the corresponding (and not necessarily optimum) values of $W$, or the reverse situation, in which the efficiency curves of $W$ are drawn, in association with the corresponding (and not necessarily optimum) values of $\delta / L$. This can be done independently for each topology or as a comparison between all of them.

The criterion related to volume, linked to self-weight and therefore to the cost of the structure, is very relevant. Indeed, this research tends to show that these structures are very stiff but are heavier than more classical structures, such as trusses. For a practical project, the criterion linked to the volume (or self-weight or quantity of materials) is thus very relevant. Moreover, our studies showed that the optimum structures in terms of volume are not very far from the optimum structures in terms of deflection.

Hence, the aim of this paper is to prove the feasibility of such footbridges. Therefore, it seems relevant and sufficient to consider the lightest structures (efficiency curves related to $W$ ) and to show that the associated values of $\delta / L$ remain acceptable. This is why the results shown in this paper focus mainly on the efficiency curves of $W$ and not of $\delta / L$.

Fig. 13 shows the efficiency curves of $\mathrm{W}$ of the footbridges composed of $\mathrm{S}$ elementary simplex modules [family S1/S2 described in Fig. 7(a)]. Fig. 13 leads to the following comments (curves $S=2$ showed bad efficiency in terms of volume and deflection and were not drawn):

- The minimum volume of materials $V_{\min }\left(\right.$ or $\left.W_{\min }\right)$ increases with the growing values of the indicator of buckling $\Psi$ and of the indicator of self-weight $\Phi$ (or the span $L$ ).

- To the contrary, the associated deflection $\delta / L$ (or $L / \delta$ ) shows small variations with $\Psi$ and $\Phi$ (in particular for $S>4$ ).

- Globally, whatever the value of $\Psi$, the minimum volume of materials $V_{\min }$ (or $W_{\min }$ ) increases with the number of elementary tensegrity modules $S$.

- $S=5$ always shows very good stiffness $L / \delta$ above 550 . Moreover, since the deflection $\delta$ is also due to self-weight, the deflection under live loads only is much better than $L / 550$. The corresponding values of $L / H$ are always between 6 and 10 .

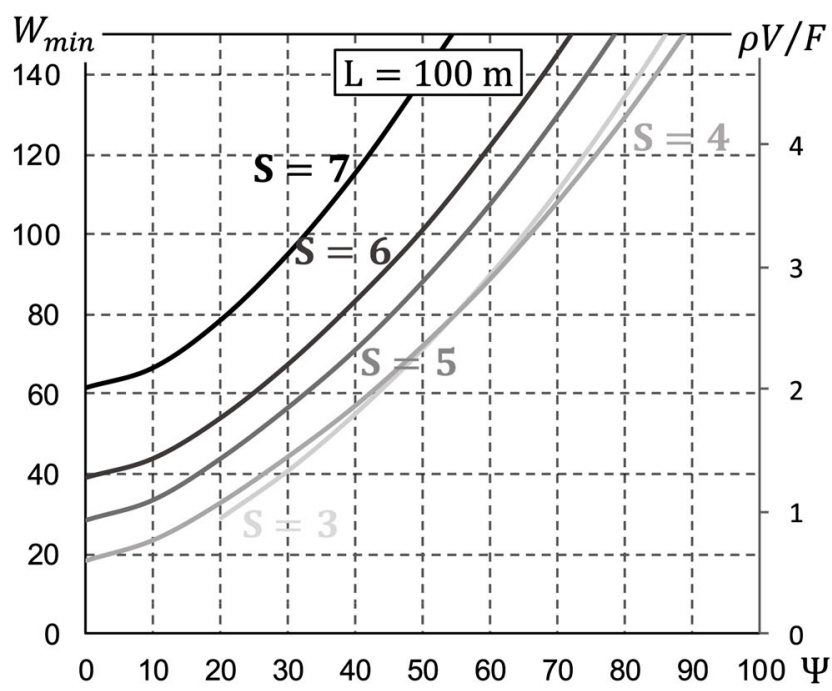

Fig. 15. Correction of Fig. 13 with constraints $\delta \leq L / 500, h_{r} \geq 2 \mathrm{~m}$, and $b \geq 2 \mathrm{~m}$ (topology S2). All other cases have been rejected. 
- $S=3$ and $S=4$ have a better stiffness $L / \delta$ than $S=5$ when $\Phi$ (or the span $L$ ) and $\Psi$ increase.

- The choice $3 \leq S \leq 5$ is always best, whatever the values of $\Psi$ and $\Phi$.

However, Fig. 13 does not exclude solutions with an insufficient stiffness $(\delta=L / 500$ is considered a maximum in this paper, as discussed in the section "Allowable Deflections"), an insufficient headroom $h_{r}$ for pedestrians, or an insufficient deck width $b$ ( $2 \mathrm{~m}$ for both seems to be a minimum). Imposing a minimum value for $h_{r}$ and $b$ is equivalent to imposing a maximum value of ratio $L / H$, noted $(L / H)_{\text {limit }}$. Fig. 13 should thus be corrected according to the methodology described in Fig. 14.

- Case (a): the solution with minimum volume corresponds to $L / \delta \geq 500$.
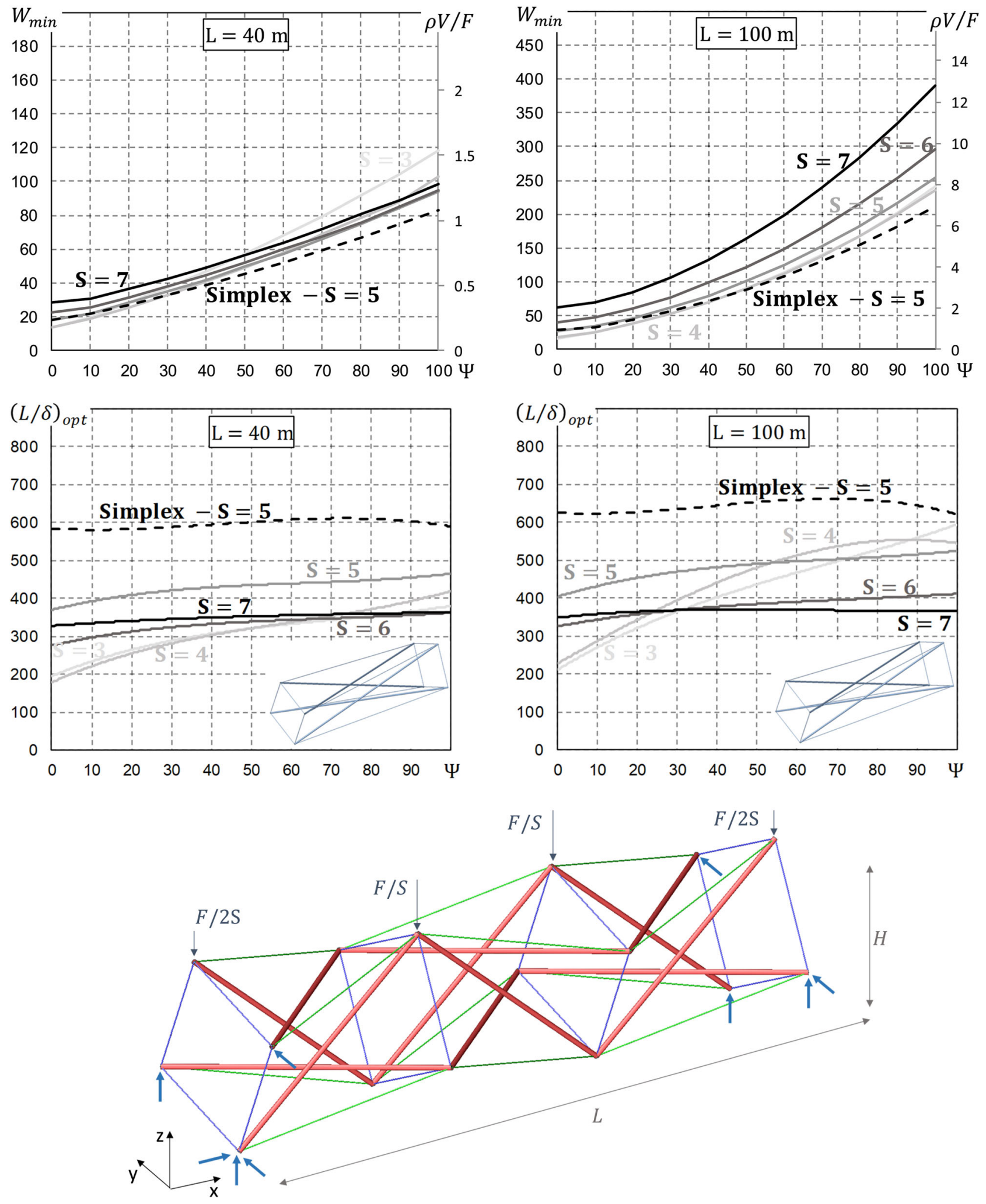

Fig. 16. Family Q1, composed of quadruplex of Type 1. 
- Cases (b) and (c): the solution with minimum volume is out of the range of sufficient stiffness. However, a solution exists with $L / \delta=500$, but a higher volume of material $W_{\min }$ is needed.

- Case (d): no solution with $L / \delta \geq 500$ exists.

- Case (e) is similar to Case (a), but a nonoptimum solution has to be considered to ensure sufficient headroom and deck width combined with $L / \delta \geq 500$.
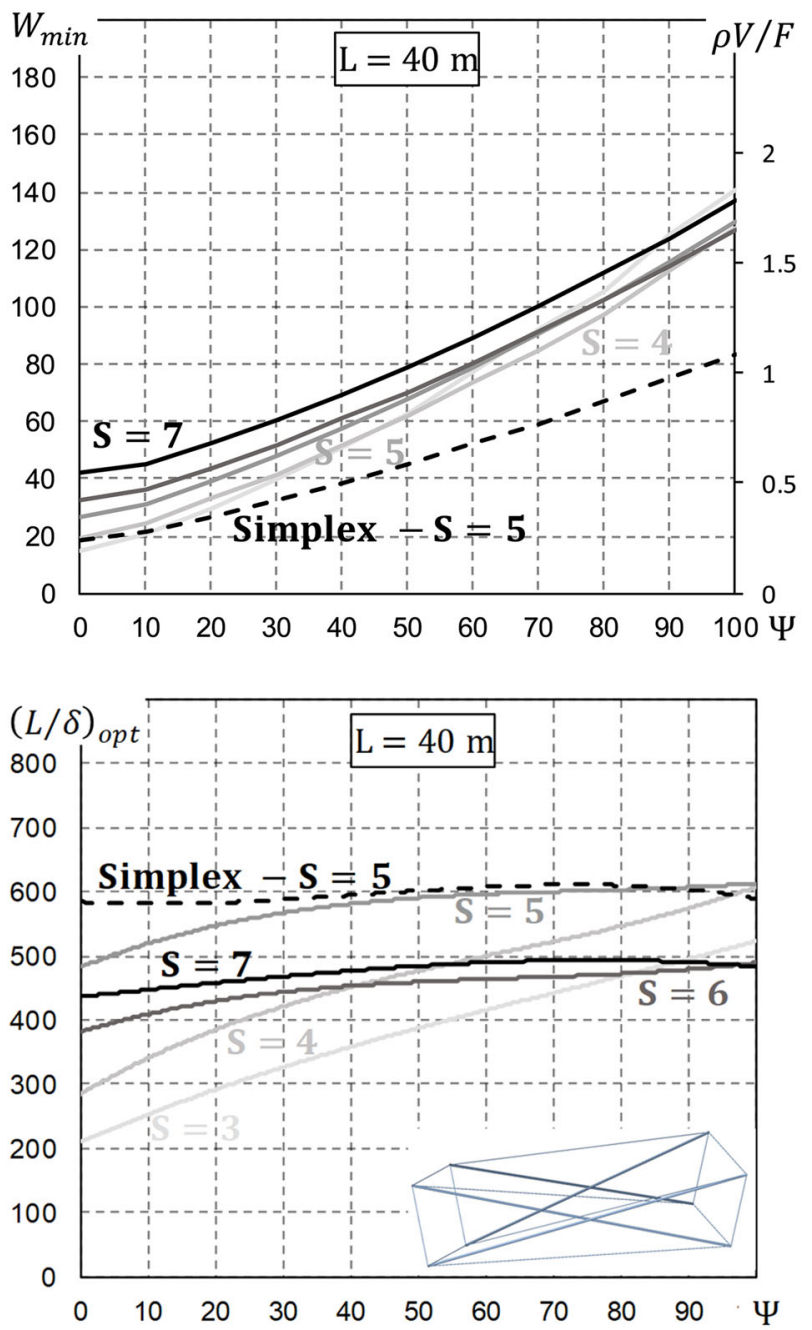

- Case (f) is similar to Case (a), but no solution can be found with the three criteria respected.

Fig. 15 is a correction of Fig. 13 according to Fig. 14 in the way that it shows, for spans equal to $40 \mathrm{~m}$ and $100 \mathrm{~m}$, respectively, the optimum structures of topology S2 combining $\delta \leq L / 500, h_{r} \geq 2 \mathrm{~m}$, and $b \geq$ $2 \mathrm{~m}$. All the other situations have been rejected. On Fig. 15 are pointed out two S2 structures related to $\Psi=48$ and, respectively, $S=4$ and
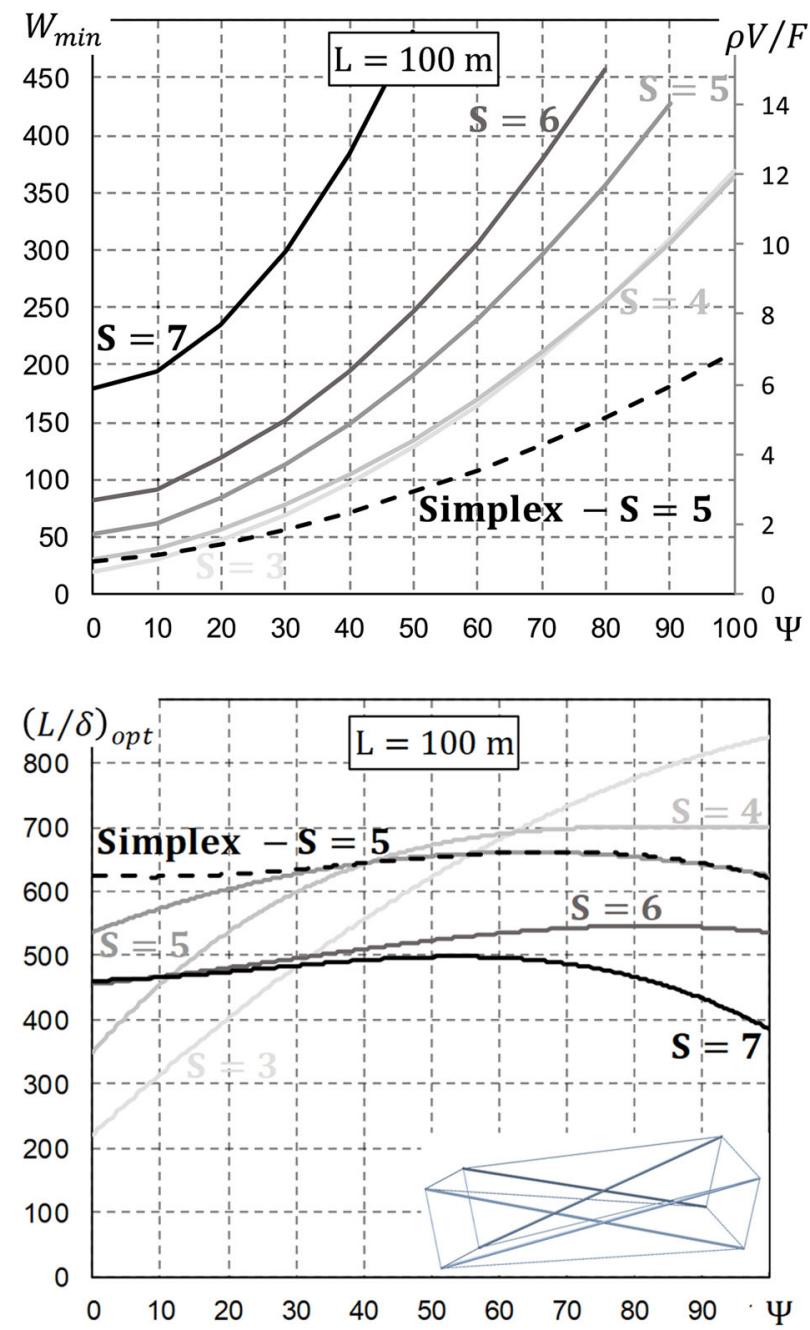

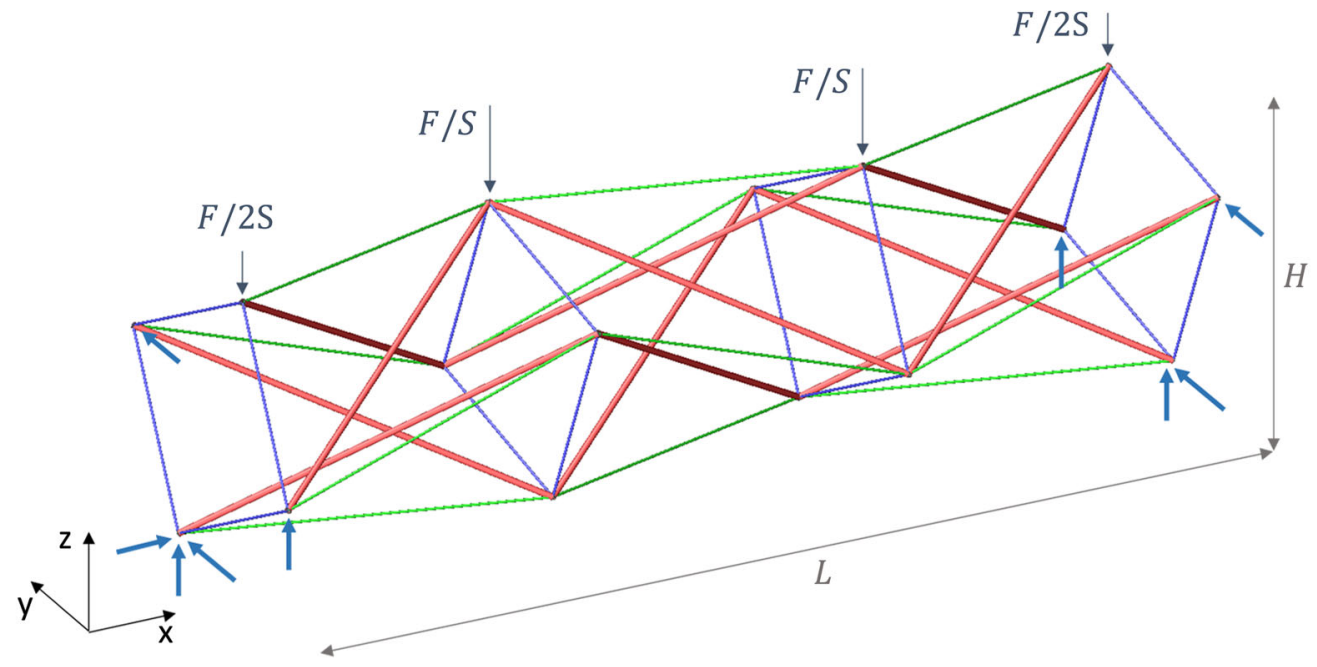

Fig. 17. Family Q2, composed of quadruplex of Type 2 . 
$S=5$. These two structures are further described in section "Practical Example: 40-m-Span Tensegrity Footbridge."

\section{Study of the Other Topologies Q1, Q2, P1, P2, H1, and H2}

While the previous section detailed the S1/S2 family, composed of simplex modules, this section summarizes the results for the other topologies. To make the comparison easier, the curve $S=5$ related
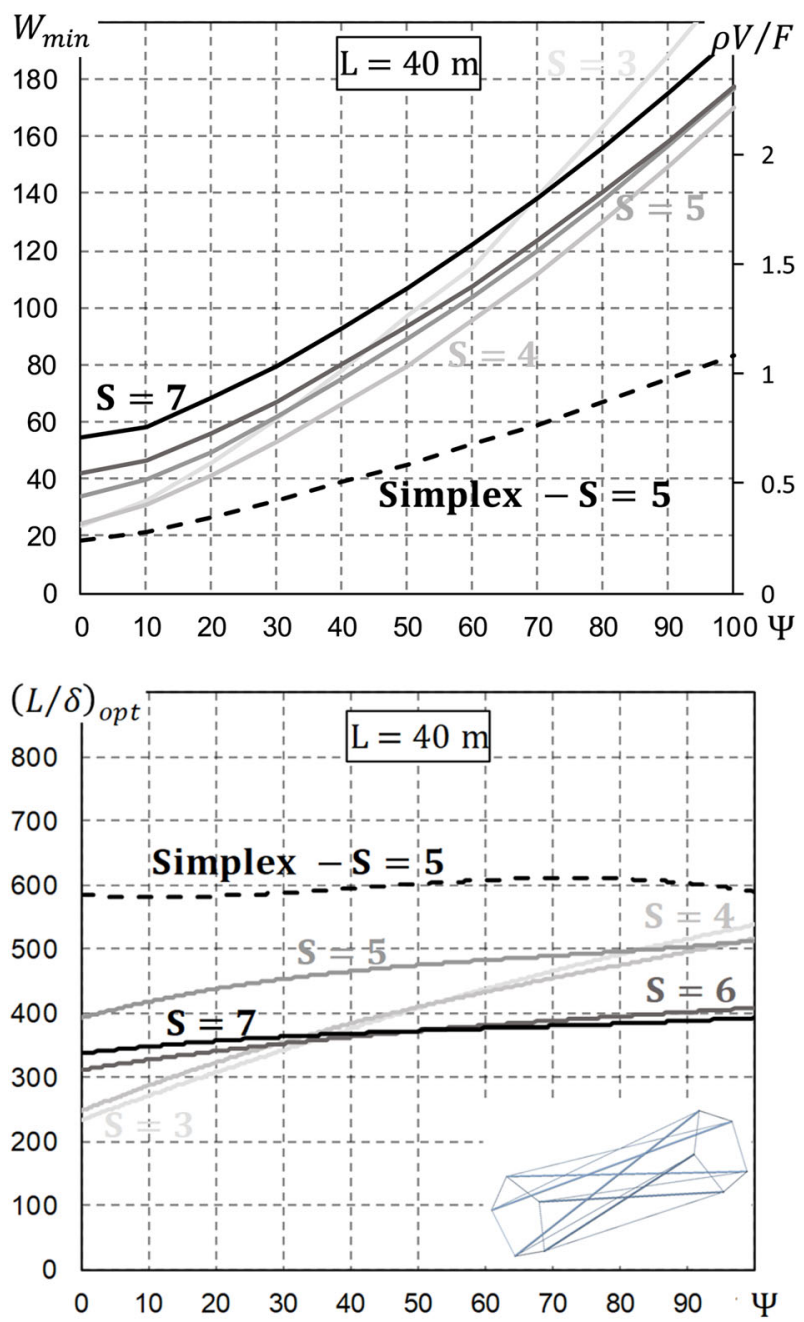

to the $\mathrm{S} 1 / \mathrm{S} 2$ topology has been systematically added to Figs. 16-21, in dashed lines. In this section, the optimum values are given with no constraint on $h_{r}$ and $b$, in contrast with the section "General Comparison of the Seven Topologies."

\section{Family Q1}

Fig. 16 shows that this family has a stiffness that seldom reaches $L / \delta=500$, while the simplex family shows a better stiffness, around $L / \delta=600$, whatever the span or the value of $\Psi$. For $S=6$
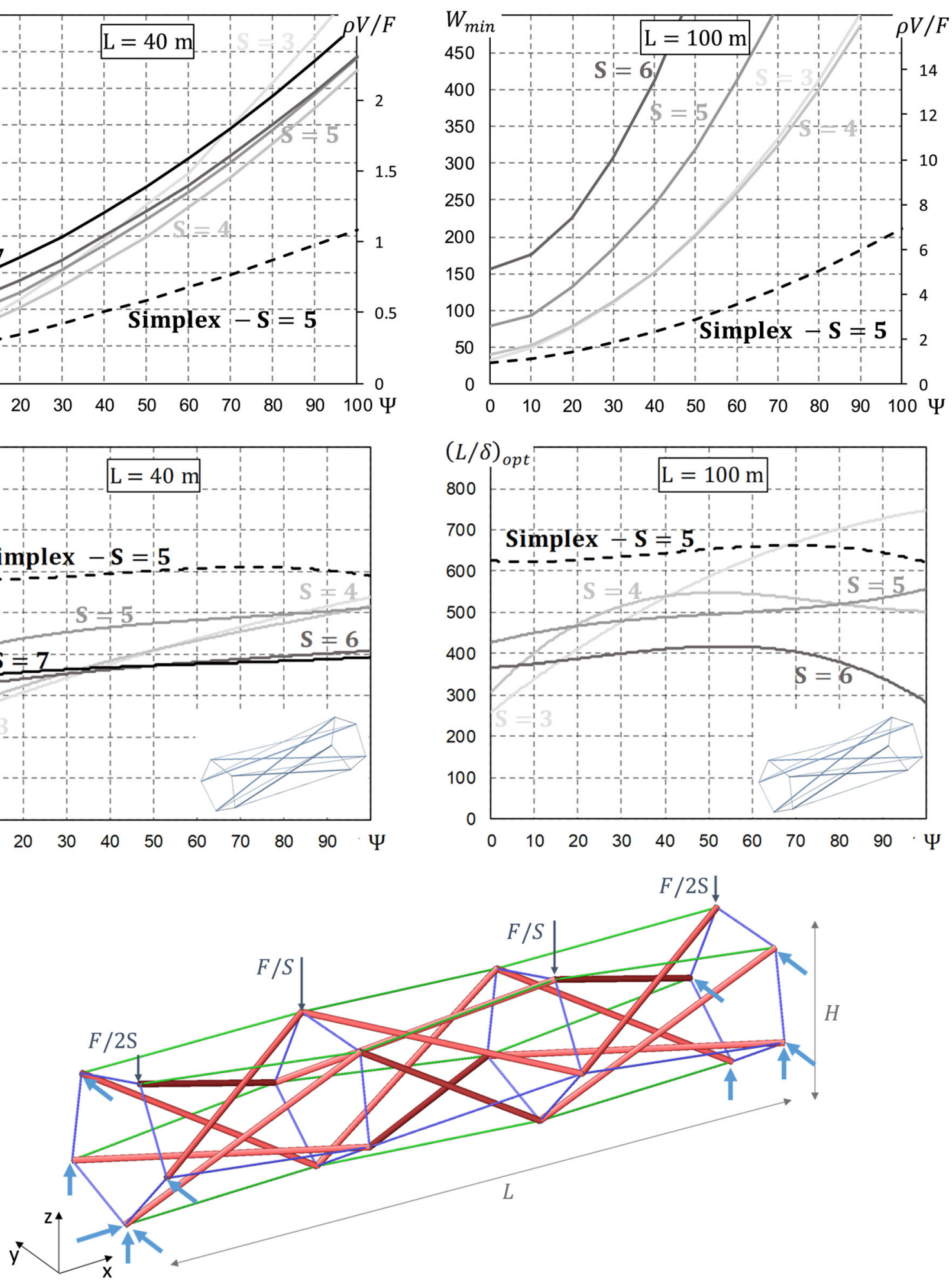

Fig. 18. Family P1, composed of pentaplex of Type 1 . 

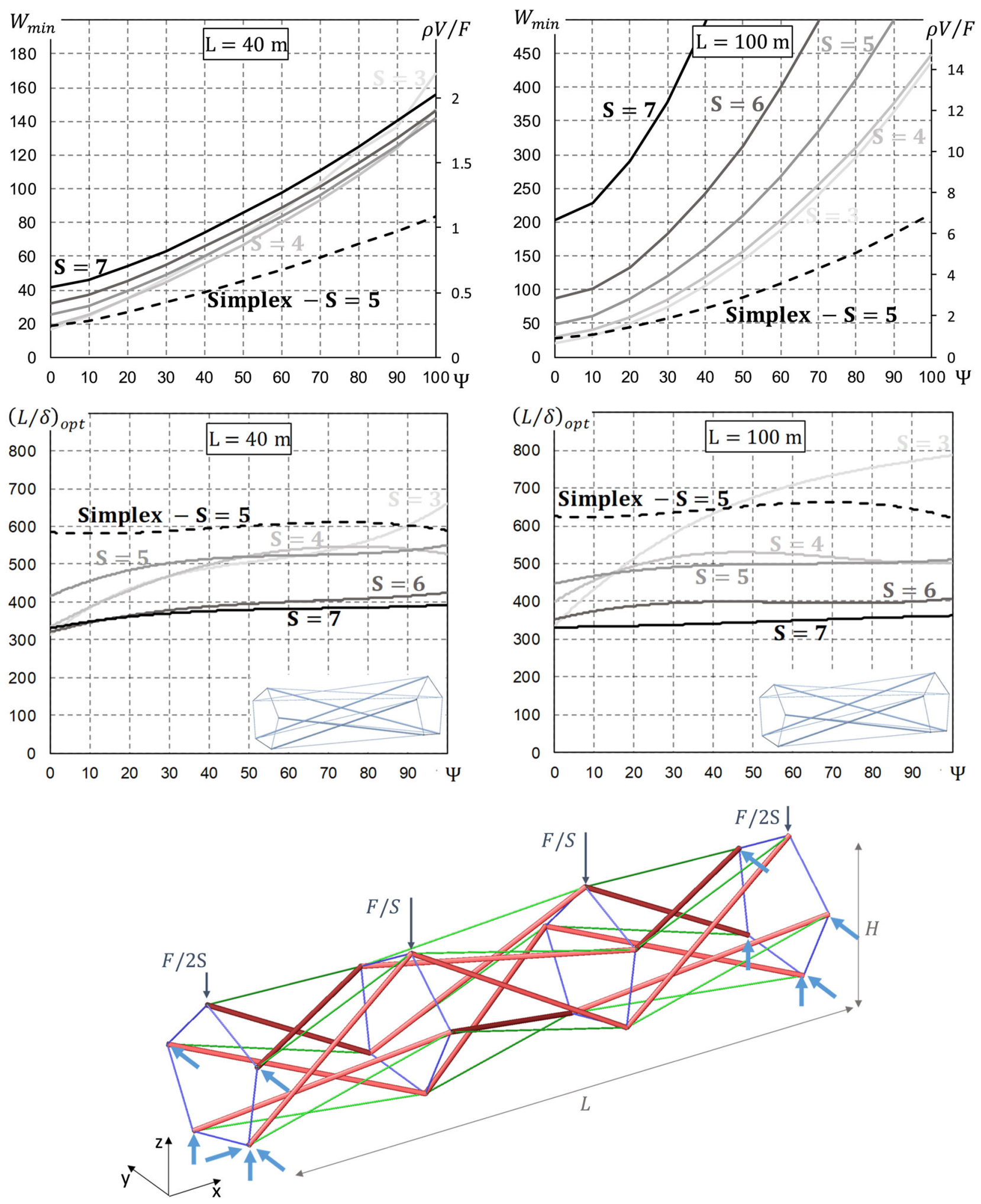

Fig. 19. Family P2, composed of pentaplex of Type 2 .

and $S=7$, the stiffness is never better than $L / \delta=400$, whatever the span and the values of $\Psi$. In terms of stiffness, $S=5$ is always better than all the others, except for large spans and large values of $\Psi$, where $S=3$ and $S=4$ can slightly overtake $L / \delta=500$.

The volume is always higher than for the simplex family, except for low values of $\Psi$, whatever the span, but only for $S=3$ and $S=4$. However, the gain in volume with respect to the simplex family is insignificant and, furthermore, small values of $\Psi$ for $S=3$ and $S=4$ correspond to a very bad stiffness.

In conclusion, excluding considerations linked to the headroom, this family of structures is never more advantageous than the simplex family, even if choices such as $S=3, S=4$ or $S=5$ for large spans and high values of $\Psi$, can lead to acceptable solutions. 

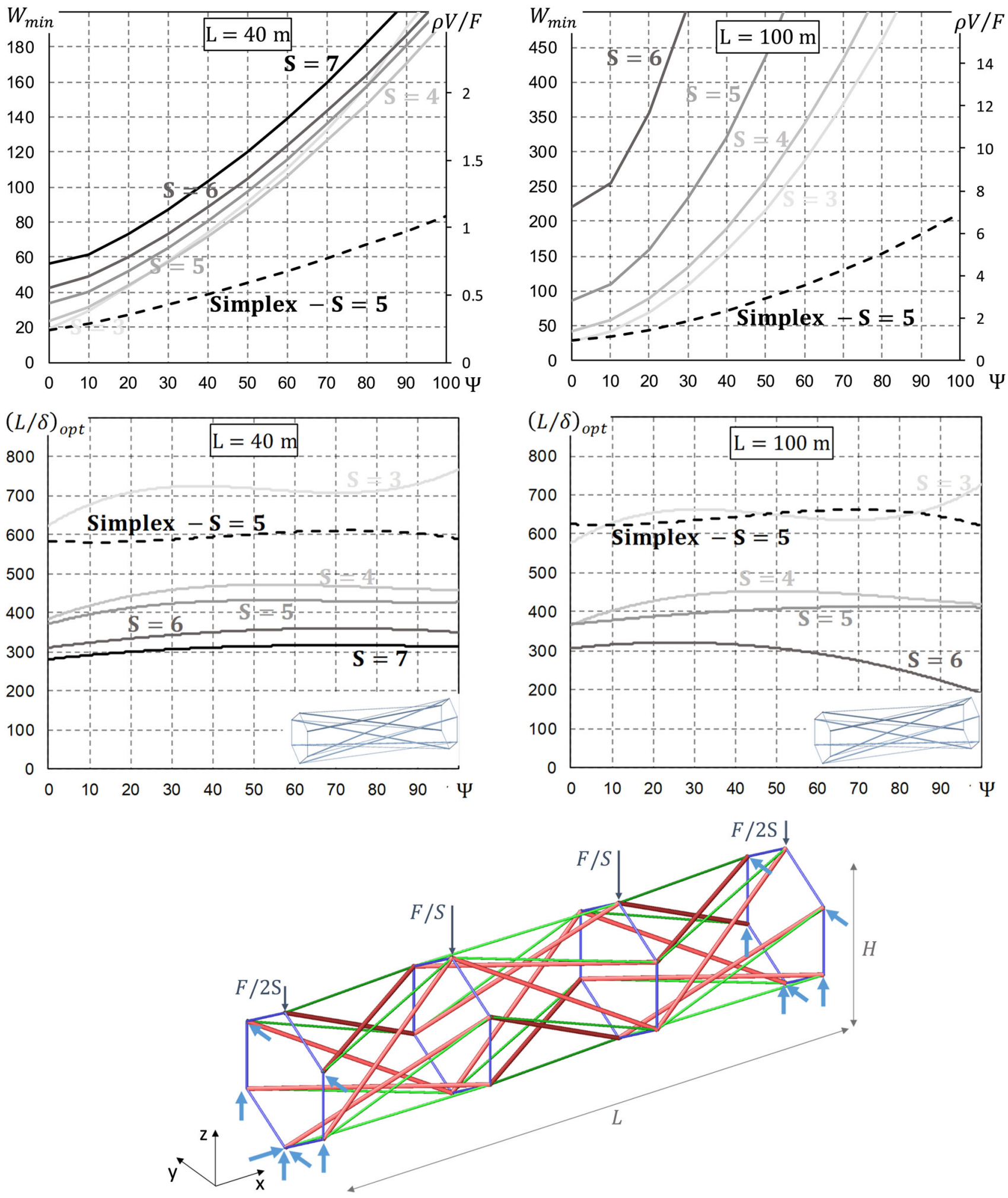

Fig. 20. Family H1, composed of hexaplex of Type 1.

\section{Family Q2}

Fig. 17 shows better behavior in the Q2 family than in the Q1 family regarding stiffness, and many solutions with deflections better than $L / 500$ can be found. In particular, the solution $S=5$ is almost always the best regarding stiffness and is equivalent to the simplex family. However, for similar performance regarding deflection, the volume of materials is much higher than for the simplex family, and the volume increases very quickly up to irrelevant values. There is an exception for $S=3$ and $S=4$ and for very small values of $\Psi$, but their lack of stiffness disqualifies them. In conclusion, this family of structures is interesting only with $S=5$ and for small values of $\Psi$, or for reasons linked to the headroom.

\section{Family P1}

Fig. 18 shows that the structures of the P1 family are always heavier than those of the simplex family, whatever the span and the value 

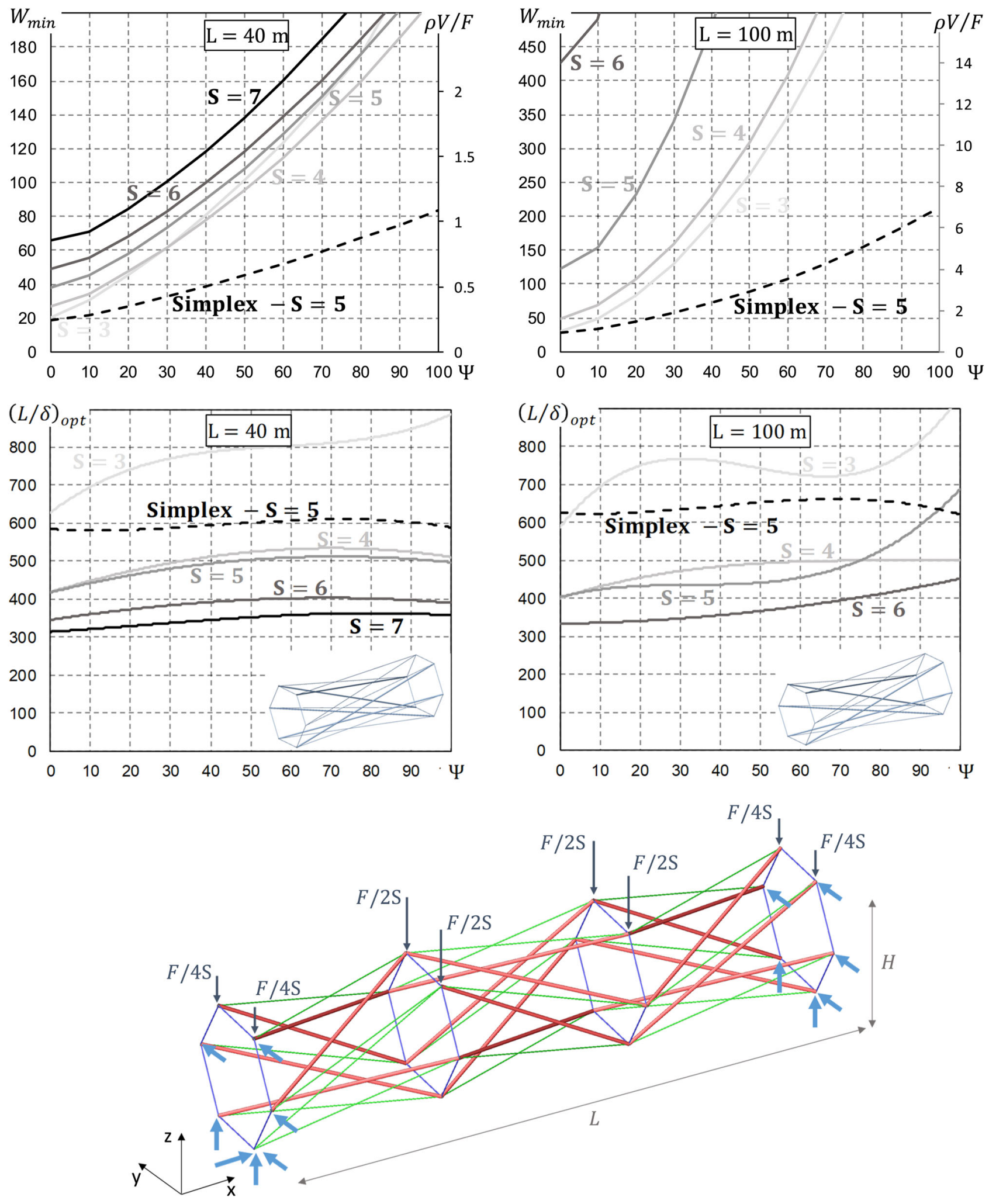

Fig. 21. Family H2, composed of hexaplex of Type 2.

of $\Psi$. For values of $\Psi$ above 50, the volume is at least doubled in comparison with the simplex family. In terms of volume, the only acceptable solution could be related to $S=4$ (and even $S=3$ ) only for values of $\Psi$ lower than 20 , but these situations correspond to a low stiffness.

The conclusion is that this family should probably be rejected for most cases.

\section{Family P2}

The results for this family are very similar to those of the P1 family, as shown in Fig. 19. In terms of stiffness, $S=3, S=4$, and $S=5$ are acceptable and slightly better than for the P1 family. However, the volume grows quickly after $\Psi=40$, leading to unreasonable solutions, particularly for large spans. 


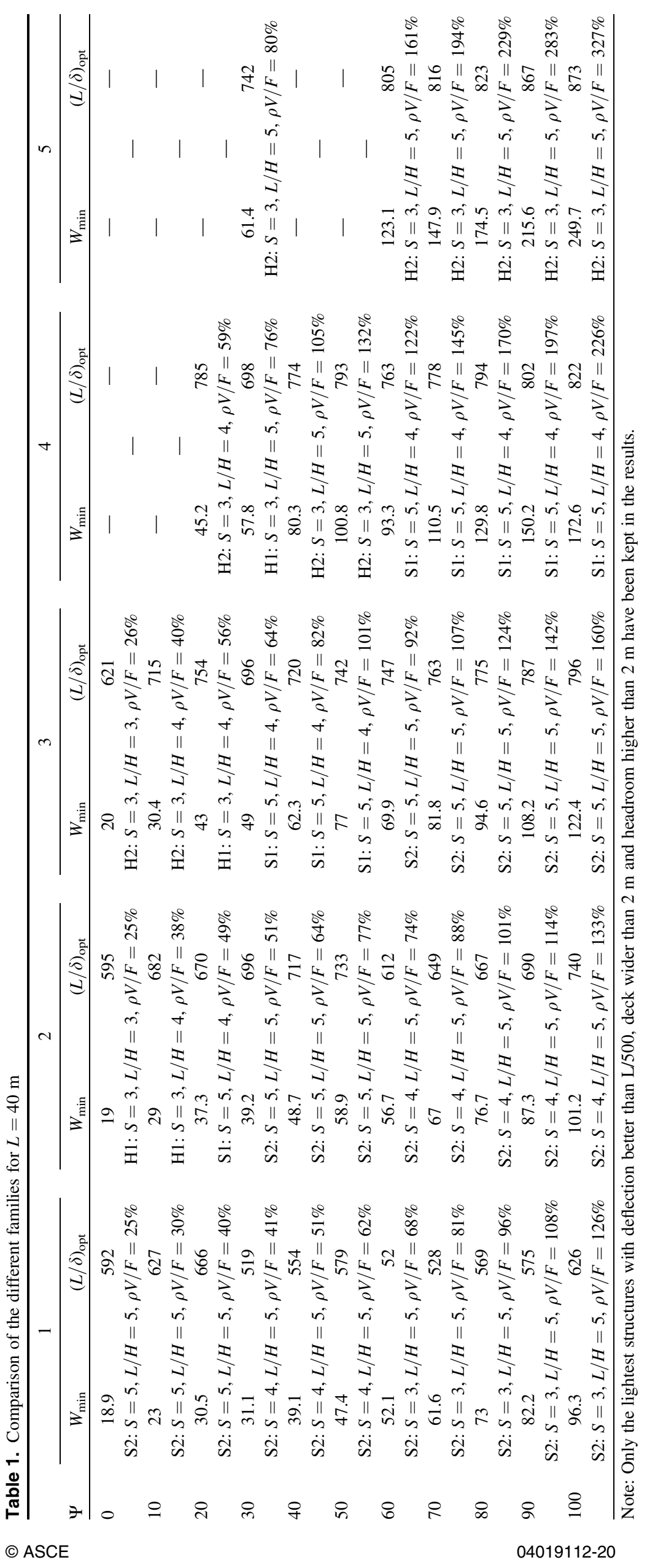




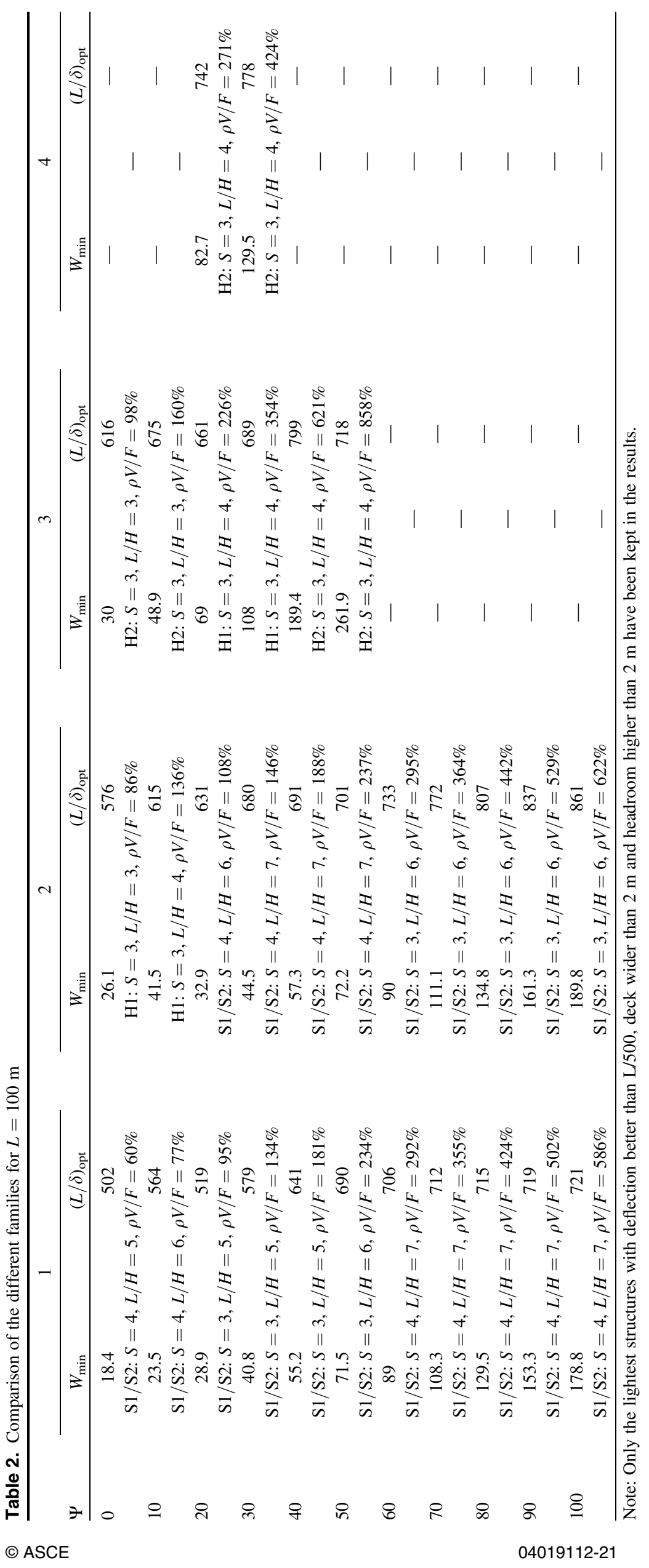



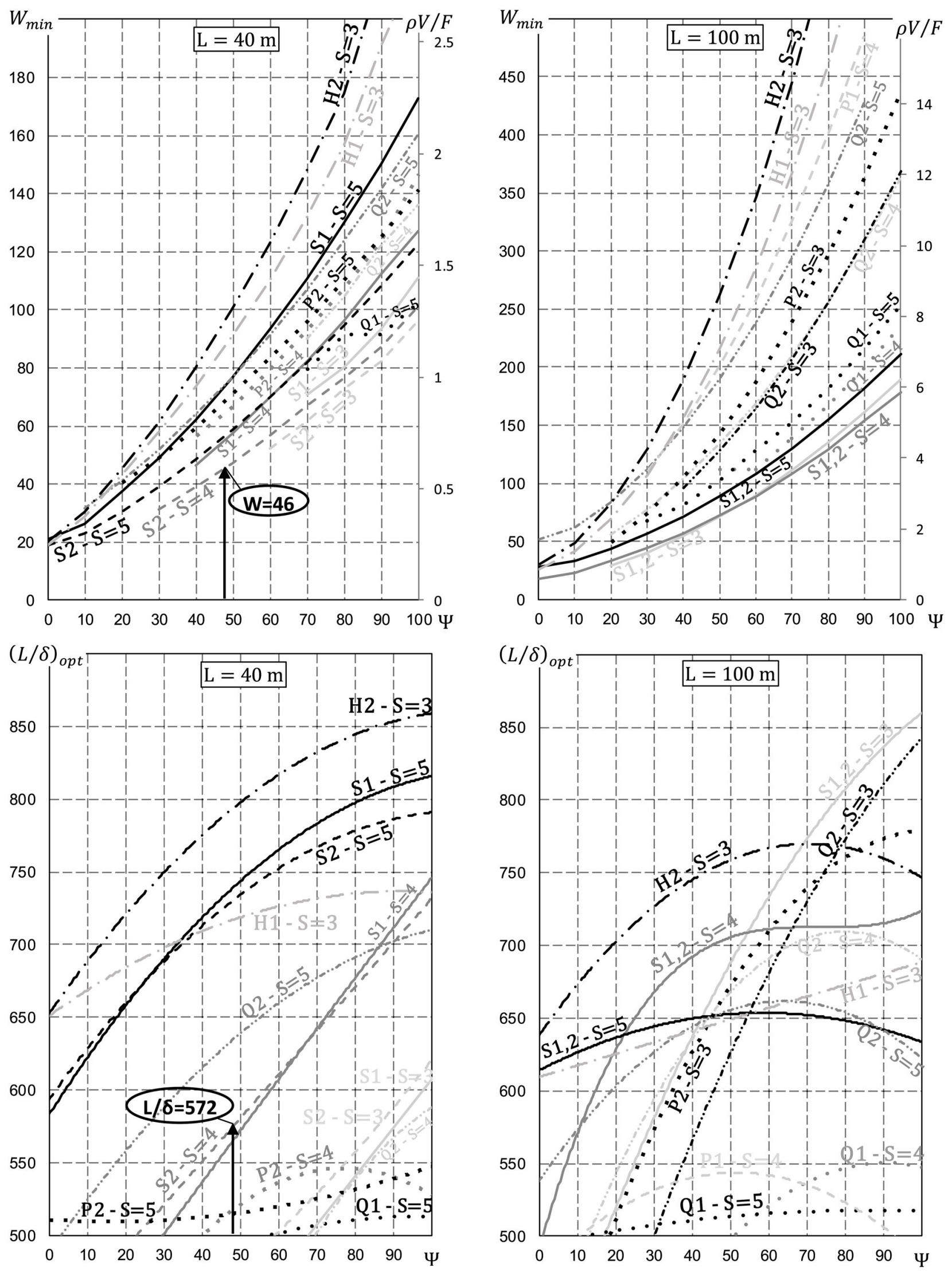

Fig. 22. Comparison of different families for $L=40 \mathrm{~m}$ and $L=100 \mathrm{~m}$. Only structures with $\delta \leq L / 500$ (deck width) and $h_{r} \geq 2 \mathrm{~m}$ (headroom for pedestrians) have been kept. 
In conclusion, excluding considerations linked to the headroom, this family of structures is never more advantageous than the simplex family, even if choices such as $S=3, S=4$, and $S=5$ for small values of $\Psi$ can lead to acceptable solutions in terms of volume, but with deflections slightly higher than L/500.

\section{Family $\mathrm{H1}$}

Fig. 20 shows that the $\mathrm{H} 1$ family is interesting for $S=3$, with good deflection performance, even sometimes better than that of the S1/S2 family. However, the volume remains much higher, particularly for values of $\Psi>30$. Its interest is therefore limited to structures with short or middle spans associated with a low value of $\Psi$; otherwise the volume, and thus the cost, becomes unreasonable. Solutions also exist with $S=4$ or $S=5$ but with a higher volume than $S=3$ and with deflections slightly higher than L/500.

\section{Family H2}

Fig. 21 shows that the $\mathrm{H} 2$ family has characteristics very similar to those of the H1 family. Similar conclusions can be drawn. $S=3$ shows excellent stiffness, always better than that of the simplex family. However, for values of $\Psi>20$, the volume grows unreasonably. In conclusion, solutions exist with $S=3, S=4$, and $S=5$, but only when $\Psi$ is small.

\section{General Comparison of the Seven Topologies}

This section brings together the results presented in the sections "Results for Family S1/S2 (Simplex)" and "Study of the Other Topologies $\mathrm{Q} 1, \mathrm{Q} 2, \mathrm{P} 1, \mathrm{P} 2, \mathrm{H} 1$, and H2" in order to make a general comparison.
Only the following structures with $\delta \leq L / 500, h_{r} \geq 2$ m (headroom for pedestrians), and $b \geq 2 \mathrm{~m}$ (deck width) have been kept:

- family S1/S2 (simplex) with $S=3, S=4$, and $S=5$,

- family Q1 (quadruplex of Type 1) with $S=4$ and $S=5$,

- family Q2 (quadruplex of Type 2) with $S=3, S=4$, and $S=5$,

- family P1 (pentaplex of Type 1) with $S=4$,

- family P2 (pentaplex of Type 2) with $S=3, S=4$, and $S=5$,

- family H1 (hexaplex of Type 1) with $S=3$, and

- family H2 (hexaplex of Type 2) with $S=3$.

Tables 1 and 2 summarize the best structures of Fig. 22 in terms of volume, with the associated stiffness $L / \delta$ and relative selfweight $\rho V / F$. Structures are sorted from Column 1 to Column 5 according to their indicators of volume $W$ in ascending order, keeping only those that show a better stiffness $L / \delta$ than the previous lighter structures.

Concerning the volume, Fig. 22 and Tables 1 and 2 suggest that - the $\mathrm{H} 2$ family with $S=3$ is always heavier than all the others,

- the simplex family with $S=3, S=4$, or $S=5$ always offers the lightest solutions, whatever the span or the value of $\Psi$,

- for small and medium spans, the simplex S2 solution is always better, and for growing values of $\Psi$, the number of tensegrity modules has to decrease progressively from $S=5$ to $S=3$,

- for large spans, the simplex S1 (or S2) with three or four modules is always the lightest solution,

- family Q1 also offers competitive solutions, whatever the span, for $S=4$ and $S=5$,

- all other families show unreasonable volumes, growing fast when the span or the value of $\Psi$ grows, and

- for the lightest structures (family S1/S2), the ratio of self-weight over total live load $\rho V / F$ is between $25 \%$ and $125 \%$ for $L=40 \mathrm{~m}$ and between $75 \%$ and $600 \%$ for $L=100 \mathrm{~m}$, depending on the values of $\Psi$.

Concerning the stiffness, Fig. 22 and Tables 1 and 2 suggest that the $\mathrm{H} 2$

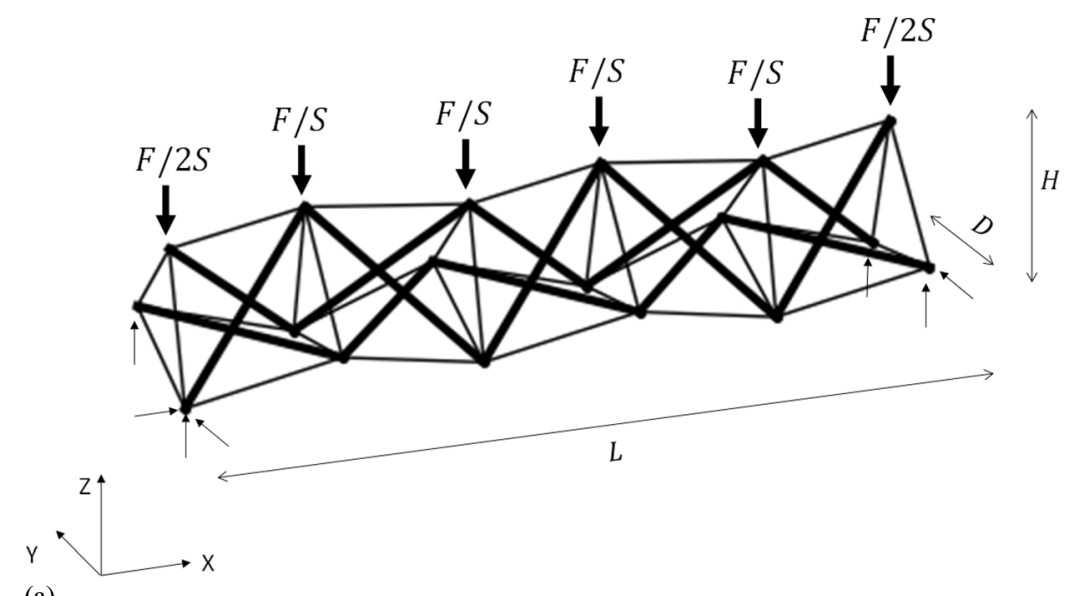

(a)
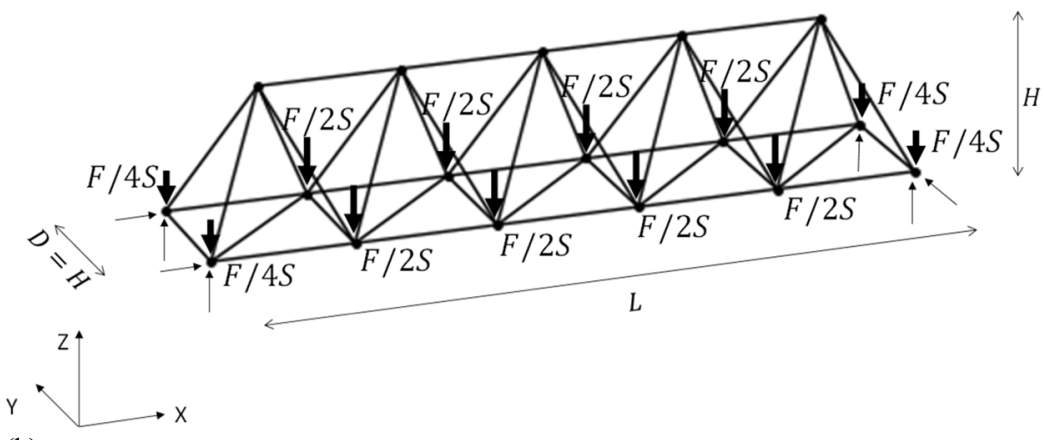

(b)

Fig. 23. Comparison between (a) tensegrity structure of topology $S 1 / \mathrm{S} 2$ with $S=5$ and (b) classical truss composed of five pyramidal modules. 
family with $S=3$ is globally always stiffer than the others. However, as noted in the preceding discussion, it is also always the heavier and should always be rejected, except for very small values of $\Psi$. According to the foregoing comments concerning the volume, if only families $\mathrm{S} 1 / \mathrm{S} 2$ and Q1 remain, one can conclude that, concerning the stiffness,

- for small and medium spans, the simplex $\mathrm{S} 1 / \mathrm{S} 2$ with $S=5$ is very good ( $L / 600$ up to $L / 800)$,

- S1/S2 with $S=4$ or $S=3$ also offer a very good stiffness for growing values of $\Psi$,

- for large spans, the simplex S1 (or S2) with three or four modules offers the stiffest solutions, and

- family Q1 with $S=4$ or $S=5$ also offers admissible solutions, especially for high values of $\Psi$.

Finally, taking into account the aspects of both volume and stiffness, the simplex family has shown its superiority, followed by the Q1 family with $S=4$ or $S=5$.

\section{Comparison between a Tensegrity Footbridge of Topology S1/S2 and a Truss Footbridge}

In this section, a tensegrity footbridge of topology S1/S2 (simplex with straight or sinusoidal deck), composed of five simplex mod- ules [Fig. 23(a)], is compared with a truss footbridge composed of five pyramidal modules [Fig. 23(b)]. The loads are uniformly distributed on the deck level of the truss, and steel is also used for each element (in such a way that all elements in tension have the same cross section and all elements in compression have the same cross section). Fig. 24 shows that for a span of $40 \mathrm{~m}$, the tensegrity structure is always about three to four times heavier $(83 / 27=3.1$ and $18 / 4=4.5)$ than the lightest truss. Cadoni and Micheletti (2012) compared the structural performance of a singlelayer tensegrity dome with a conventional truss dome and got similar results. This important result is discussed in the conclusion and must be nuanced. The truss is also slightly stiffer and corresponds to lower values of $L / H$; in other words, the tensegrity structure has to be more slender than the truss to reach an optimum self-weight.

\section{Practical Example: 40-m-Span Tensegrity Footbridge}

The assumptions are the following:

- span $L=40 \mathrm{~m}$,

- deck width $b=2 \mathrm{~m}$,
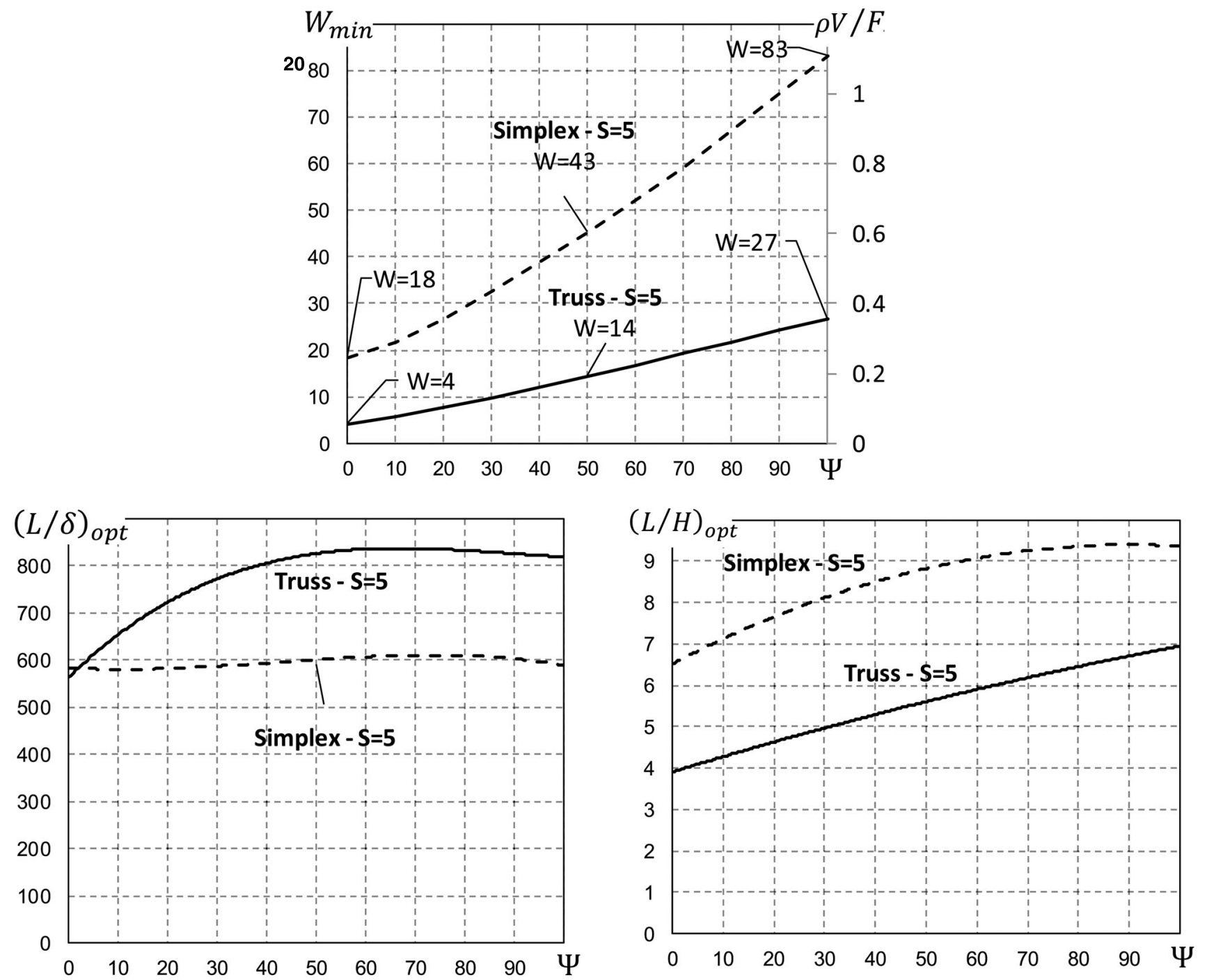

Fig. 24. Comparison between efficiency curves of $W$ for a truss with $S=5$ and for a simplex topology $\mathrm{S} 1 / \mathrm{S} 2$ with $S=5$ for $L=40 \mathrm{~m}$ and $\Phi=125 \times 10^{-4}$, regardless of any constraints related to the deck's width or headroom. 
- struts have hollow circular cross sections with a ratio $e / d$ equal to 0.1 , which means a factor $q$ equal to 0.363 (Fig. 8),

- cables have full cross sections,

- all materials are S355 steel $(\sigma=237 \mathrm{MPa}, E=210.000 \mathrm{MPa}$, $\left.E / \sigma=886, \rho=77 \mathrm{kN} / \mathrm{m}^{3}\right)$,

- dead loads related to the deck are estimated at $1 \mathrm{kN} / \mathrm{m}^{2}$, and live loads acting on the deck, including snow and wind, are estimated at $5.5 \mathrm{kN} / \mathrm{m}^{2}$; this corresponds to a total load $F=(1+5.5) \times$ $2 \times 40=520 \mathrm{kN}$, and

- the indicator of buckling is equal to $\Psi=\sigma_{s} L /\left(q E_{s} F\right)^{1 / 2}=48$, and the indicator of self-weight is equal to $\Phi=\rho L / \sigma=0.0130$.

Fig. 25 shows a few good possible solutions, in terms of both volume and/or stiffness, and considering headroom aspects. The lightest solution, Fig. 25(a), corresponds to the structure made of simplex S2 (snake deck) with $S=4, W=46$, and $L / \delta=572$ (the corresponding point is illustrated in Fig. 15 and 22). The selfweight is about $7.8 \mathrm{kN} / \mathrm{m}$, which is not so unusual for footbridges. The example shows the benefit of the sinusoidal deck compared with the straight deck: $311 \mathrm{kN}$ instead of $372 \mathrm{kN}$ for the topology S1 with $S=4$ [Fig. 25(b)]. Regarding stiffness, the best structure is $\mathrm{H} 2$ with $S=3$ [Fig. 25(e)], which reaches a value of $L / \delta=786$, but for a self-weight that grows up to $16.2 \mathrm{kN} / \mathrm{m}$, that is, double that of the S2 with $S=4$ structure [Fig. 25(a)]. The structure H1 with $S=3$ [Fig. 25(d)] is not really interesting because its volume is almost as high as that of $\mathrm{H} 2$ with $S=3$ [Fig. 25(e)], for a deflection similar to that of S2 with $S=5$ [Fig. 25(c)].

Note, however, the following:

- The structure in Fig. 25(b), composed of simplex S1 (straight deck) with $S=4$, offers a good solution, with $W=55, L / \delta=561$, and headroom $2.9 \mathrm{~m}$, mainly because it corresponds to the lightest structure with a straight deck.

- The structure in Fig. 25(c), composed of simplex S2 (snake deck) with $S=5$, also offers a good solution, with $W=57$, $L / \delta=731$, and headroom $5.2 \mathrm{~m}$.
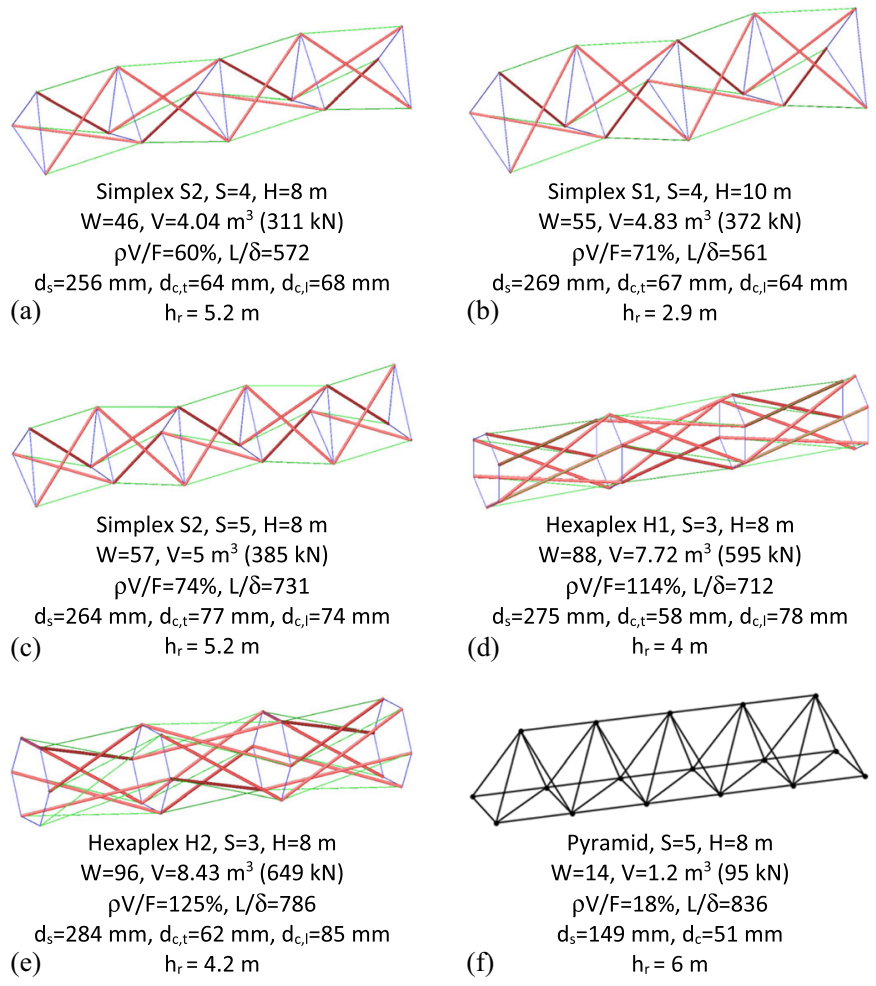

Fig. 25. Best solutions in terms of volume and/or deflection for the given practical example and considering $b=2 \mathrm{~m}$ and $\mathrm{hr} \geq 2 \mathrm{~m}$.
- The last structure, Fig. 25(f), is a truss composed of five pyramidal modules, as presented in the previous section. Its stiffness is globally slightly better than that of the tensegrity structures $(L / \delta=836)$. However, its self-weight is much lower, $W=14$, compared with that of the best tensegrity structure, $W=46$. The lightest tensegrity structures are thus, here, more than three times heavier than the truss. This result is discussed in the conclusion and must be nuanced.

Finally, Fig. 26 shows the lightest structure with no restriction on headroom and deck width: here, imposing $b \geq 2$ increases the mass by $11 \%$ in comparison with Fig. 25(a), the simplex S2 with $S=4$ related to $W=46$.

The severity of the dynamic interaction with walking pedestrians is assessed by means of a modal analysis. Table 3 summarizes the natural frequencies computed for both the $\mathrm{S} 2$ tensegrity structure with $S=4$ (Fig. 26) and the truss of Fig. 25(f). They are calculated with the tangent stiffness matrix of the structure, considering the self-weight load case and the lumped mass matrix. The first three torsional modes are much lower for the tensegrity structure $(0.15,0.34$, and $0.45 \mathrm{~Hz})$ than for the truss $(32.0,59.5$, and $99.7 \mathrm{~Hz}$ ), which confirms the greater deformability of the former system in torsion, as well as an increased mass compared with that of the truss. The fundamental bending mode in the vertical plane is above $30 \mathrm{~Hz}$, which confirms the very high stiffness of the tensegrity structure in the vertical direction. More important, the bending mode in the vertical direction and the torsional modes-which have a component in the vertical direction-are outside the main critical range corresponding to the vertical dynamic loading of walking pedestrians, that is, 1.7$2.1 \mathrm{~Hz}$; quite far outside, in fact. Furthermore, in the horizontal direction, only torsional modes-which have a component in the

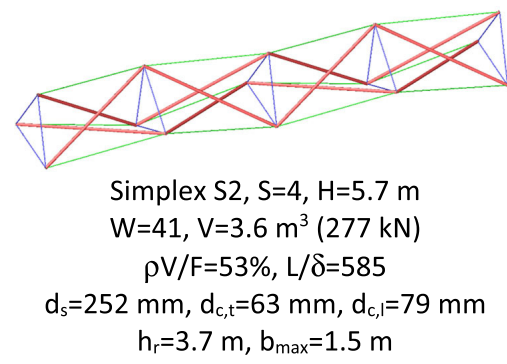

Fig. 26. Lightest tensegrity structure composed of prismatic modules for the given practical example regardless of deck's width and headroom aspects.

Table 3. Natural frequencies computed for tensegrity structure (simplex S2) and truss

\begin{tabular}{|c|c|c|}
\hline Mode No. & Frequency $(\mathrm{Hz})$ & Description \\
\hline \multicolumn{3}{|c|}{ Simplex S2: $S=4, H=5.7 \mathrm{~m}$} \\
\hline 1 & 0.15 & First torsional vibration mode \\
\hline 2 & 0.34 & Second torsional vibration mode \\
\hline 3 & 0.45 & Third torsional vibration mode \\
\hline 4 & 30.2 & First bending mode in vertical plane \\
\hline 5 & 55.9 & First bending mode in horizontal plane \\
\hline \multicolumn{3}{|c|}{ Truss: $S=5, H=8 \mathrm{~m}$} \\
\hline 1 & 1.09 & Shear deformation in horizontal plane \\
\hline 2 & 26.0 & First swaying mode \\
\hline 3 & 32.0 & First torsional vibration mode \\
\hline 4 & 59.5 & Second torsional vibration mode \\
\hline 5 & 99.7 & Third torsional vibration mode \\
\hline
\end{tabular}


horizontal direction too-need to be considered. Their natural frequencies also lie outside the main critical range for resonance with human walk, that is, $0.5-1.1 \mathrm{~Hz}$. This is not the case for the truss structure, which features a horizontal mode shape at $1.09 \mathrm{~Hz}$. This problem could be fixed by changing the support conditions of the truss, but this goes beyond the scope of this paper. Finally, it is also noticed that the more important mass of the tensegrity structure makes it less prone to human-structure synchronization (Sétra 2006) and vandalism excitation (Schwartz et al. 2013).

\section{Discussion and Conclusion}

As noted in the abstract, the architectural potential of tensegrity structures is proven, yet, paradoxically, very few real construction projects have sprung up around the world. This fact has motivated the authors to prove the practical feasibility of such structures. This work first needed the important research summarized in Latteur et al. (2017), which offered the possibility of using a rigorous design and optimization methodology based on morphological indicators. Thanks to this design methodology, it has been possible to select the stiffest and the lightest footbridge structures while also considering design constraints such as headroom and deck width, in order to prove, or on the contrary to reject, the feasibility of some topologies. This paper highlights the good performance of several topologies, in particular the simplex family $\mathrm{S} 1 / \mathrm{S} 2$, which often reach a stiffness better than $L / \delta=600$ when subjected to both live loads and self-weight (which means excellent performance under live loads only).

The results presented in this paper rely on several assumptions detailed in the section "Assumptions and Definitions." The practical case developed in the previous section shows that even if the results in terms of stiffness and dynamic behavior are unexpectedly excellent for some topologies, the volume of such structures remains higher than the volume related to more classical structures such as trusses. However, the pyramidal truss is among the lightest structures for crossing a span. A comparison with other kinds of structures composed of bended beams or nonfunicular arches (which is often an architect's choice) would certainly lead to much heavier structures than the truss, much more similar to tensegrity structures in terms of material consumption. For instance, considering again the example of the previous section, but this time using two steel beams to cross the span and support the deck, a solution could be two HL1000x554 steel profiles, with a stiffness of $L / 476$ and a total self-weight equal to $444 \mathrm{kN}$ (deck not included), which means $11.1 \mathrm{kN} / \mathrm{m}$. This value has to be compared with the $7.8 \mathrm{kN} / \mathrm{m}$ for the lightest tensegrity structure. Furthermore, the fundamental frequency of the beams would be equal to $2.1 \mathrm{~Hz}$, which is inside the critical range for pedestrians. This simple example proves that the tensegrity structure is not necessarily a solution to avoid and is not necessarily too heavy when compared with other kinds of structures or special architectural structures.

Furthermore, there exist several tracks for investigation in order to reduce the self-weight of tensegrity structures used as footbridges.

- Materials-The efficiency curves of $W$ presented in this paper are related to steel with $E_{s} / \sigma_{s}=E_{c} / \sigma_{c}=886$ and $\sigma_{s}=\sigma_{c}=$ $237 \mathrm{MPa}$. However, for cables, other mechanical characteristics could be considered, for instance $\sigma_{c}=1,000 \mathrm{MPa}$ and $\mathrm{E}_{c} / \sigma_{c}=$ 210 , while struts are designed as before with $\mathrm{E}_{s} / \sigma_{s}=886$. Considering again the example of Fig. $26[S=4$ and $L / H=$ $40 / 5.7=7$ ], redrawn in Fig. 27(a), Fig. 27(c) shows that decreasing the ratio $E_{c} / \sigma_{c}$ involves a lower self-weight $(W=31)$ but also a lower stiffness $(L / \delta=175)$. It thus seems relevant to cleverly tune the material properties $(E, \sigma$, and $\rho)$ in order to minimize the self-weight while ensuring sufficient stiffness. However, it remains difficult to compare structures with different materials used for the struts, insofar as $W$ itself depends on factors $E_{s} / \sigma_{s}$ and on the indicator of buckling $\Psi$, according to Eq. (4). However, this limitation disappears if the structures have a linear behavior, as explained in the conclusion of Latteur et al. (2017).

- Prestress level-This paper assumes that the prestress level $\beta=\beta_{\min }$ is fixed at its minimum, in such a way that no cable slacks. Fig. 27(d) shows that the stiffness can be improved $(L / \delta=1,129)$ by increasing the prestress level or, in other words, by considering $\theta>1$ (with $\beta=\theta \beta_{\min }$ ). However, this also has a negative impact on the self-weight $(W=69$ for $\theta=2)$. On the contrary, considering no prestress $(\theta=0)$ with

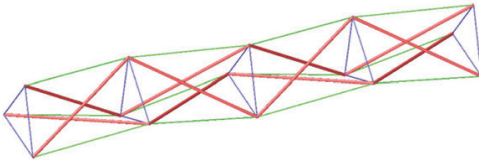

Simplex, $\mathrm{S}=4, \mathrm{H}=5.7 \mathrm{~m}$ $\mathrm{E}_{\mathrm{s}} / \sigma_{\mathrm{s}}=886, \mathrm{E}_{\mathrm{c}} / \sigma_{\mathrm{c}}=886, \theta=1$ $\mathrm{W}=41, \mathrm{~V}=3.6 \mathrm{~m}^{3}(277 \mathrm{kN})$ $\rho V / F=53 \%, L / \delta=585$ $\mathrm{d}_{\mathrm{s}}=252 \mathrm{~mm}, \mathrm{~d}_{\mathrm{c}, \mathrm{t}}=63 \mathrm{~mm}, \mathrm{~d}_{\mathrm{c}, \mathrm{l}}=79 \mathrm{~mm}$

(a)

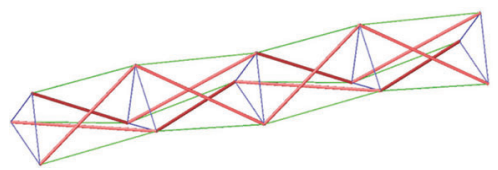

Simplex, $\mathrm{S}=4, \mathrm{H}=5.7 \mathrm{~m}$

$E_{s} / \sigma_{s}=886, E_{c} / \sigma_{c}=210, \theta=1$

$\mathrm{W}=31, \mathrm{~V}=2.7 \mathrm{~m}^{3}(209 \mathrm{kN})$

$\rho \mathrm{V} / \mathrm{F}=40 \%, \mathrm{~L} / \delta=175$

$\mathrm{d}_{\mathrm{s}}=247 \mathrm{~mm}, \mathrm{~d}_{\mathrm{c}, \mathrm{t}}=29 \mathrm{~mm}, \mathrm{~d}_{\mathrm{c}, \mathrm{l}}=37 \mathrm{~mm}$

(c)

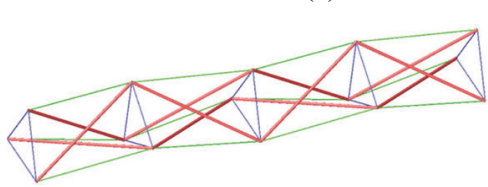

Simplex, $\mathrm{S}=4, \mathrm{H}=5.7 \mathrm{~m}$

$\mathrm{E}_{\mathrm{s}} / \sigma_{\mathrm{s}}=886, \mathrm{E}_{\mathrm{c}} / \sigma_{\mathrm{c}}=886, \theta=2$

$\mathrm{W}=69, \mathrm{~V}=6.1 \mathrm{~m}^{3}(466 \mathrm{kN})$

$\rho V / F=90 \%, L / \delta=1129$

$\mathrm{d}_{\mathrm{s}}=318 \mathrm{~mm}, \mathrm{~d}_{\mathrm{c}, \mathrm{t}}=91 \mathrm{~mm}, \mathrm{~d}_{\mathrm{c}, \mathrm{l}}=112 \mathrm{~mm}$

(d)

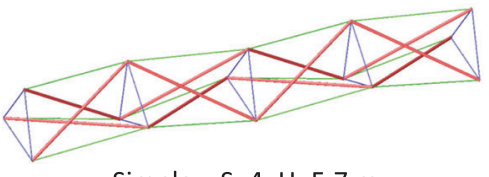

Simplex, $\mathrm{S}=4, \mathrm{H}=5.7 \mathrm{~m}$

$E_{s} / \sigma_{s}=886, E_{c} / \sigma_{c}=886, \theta=0$

$\mathrm{W}=59, \mathrm{~V}=5.2 \mathrm{~m}^{3}(399 \mathrm{kN})$

$\rho V / F=77 \%, L / \delta=297$

$\mathrm{d}_{\mathrm{s}}=206 \mathrm{~mm}, \mathrm{~d}_{\mathrm{c}, \mathrm{t}}=99 \mathrm{~mm}, \mathrm{~d}_{\mathrm{c}, \mathrm{l}}=165 \mathrm{~mm}$

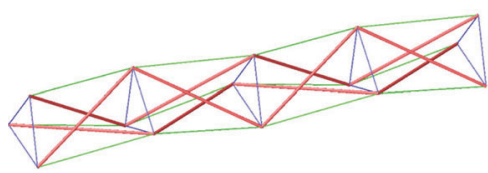

Simplex, $\mathrm{S}=4, \mathrm{H}=5.7 \mathrm{~m}$

$\mathrm{E}_{\mathrm{s}} / \sigma_{\mathrm{s}}=886, \mathrm{E}_{\mathrm{c}} / \sigma_{\mathrm{c}}=210, \theta=2$

$\mathrm{W}=49, \mathrm{~V}=4.3 \mathrm{~m}^{3}$ (331 kN)

$\rho V / F=64 \%, L / \delta=292$

$\mathrm{d}_{\mathrm{s}}=306 \mathrm{~mm}, \mathrm{~d}_{\mathrm{c}, \mathrm{t}}=41 \mathrm{~mm}, \mathrm{~d}_{\mathrm{c}, \mathrm{l}}=50 \mathrm{~mm}$

(e)

Fig. 27. Influence of material stiffness indicator $E / \sigma$ and prestress level $\beta=\theta \beta_{\min }$ on stiffness $L / \delta$ and self-weight $W$ of a structure $(S$ and $L / H$ are fixed). 
cables replaced by struts designed according to Eq. (2) leads to structures with very low efficiency in terms of both self-weight and stiffness, as shown in Fig. 27(b) $(L / \delta=297, W=59$ for $\theta=0$ ). Finding a compromise between the material stiffness indicators $E / \sigma$ and the prestress level $\beta$, both having an influence on the stiffness and the self-weight [Fig. 27(e)], would therefore be an interesting investigational path.

- Optimization of the prestress state-As explained in the section "External Loads, Self-Weight, Prestress, and Security Coefficients," one of the main assumptions of the design and optimization methodology relies on the choice of a prestress state, which may not lead to optimum structures. In other words, researching a way to insert the optimization of the prestress state directly into the design methodology seems to be a relevant, and perhaps promising, research field.

Finally, other aspects linked to robustness and to practical construction should be considered before any real construction.

\section{Acknowledgments}

The authors would like to thank BESIX Group for supporting this work. Project funded by Brussels Capital Region-Innoviris.

\section{Notation}

The following symbols are used in this paper:

$A=$ cross-sectional area;

$A_{c}, A_{c, i}=$ cross-sectional area of cable of index $i$;

$A_{c, l}=$ cross-sectional area of longitudinal cable (horizontal direction);

$A_{c, t}=$ cross-sectional area of transversal cable (triangular or polygonal bases);

$A_{s}, A_{s, i}=$ cross-sectional area of strut of index $i$;

$b=$ width of suspended deck;

$D=$ width of structure before application of external load $\tilde{\mathbf{F}}$;

$D_{r}=$ degree of redundancy of structure;

$d=$ diameter of cross section;

$d_{c, l}=$ diameter of cross section of longitudinal cable;

$d_{c, t}=$ diameter of cross section of transversal cable;

$d_{s}=$ diameter of cross section of strut;

$E=$ Young's modulus;

$E_{c}=$ Young's modulus of cable material;

$E_{s}=$ Young's modulus of strut material;

$e=$ steel thickness for hollow section;

$F=$ see definition of $\tilde{\mathbf{F}}$;

$\tilde{\mathbf{F}}=$ vector defined in section "External Loads, SelfWeight, Prestress, and Security Coefficients";

$F_{\text {pre }}=$ value of "equivalent" prestress into cable or strut of

$F_{\text {pre }, i} \quad$ index $i$, considered external axial load acting at both extremities of cable ( $>0$ because in traction) or strut $(<0$ because in compression);

$\tilde{\mathbf{F}}_{\text {pre }}=$ vector defined in section "External Loads, SelfWeight, Prestress, and Security Coefficients";

$f=$ undefined function;

$\tilde{\mathbf{f}}=$ vector defined in section "External Loads, SelfWeight, Prestress, and Security Coefficients"

$\tilde{\mathbf{f}}_{\text {pre }}=$ vector defined in section "External Loads, SelfWeight, Prestress, and Security Coefficients";

$\tilde{\mathbf{G}}=$ self-weight of structure, considered acting as external load case in combination with external load $\tilde{\mathbf{F}}$;

$H=$ height of structure;
$\mathrm{H} 1$ = family of structures composed of hexaplex of Type 1;

$\mathrm{H} 2$ = family of structures composed of hexaplex of Type 2;

$h_{r}=$ available headroom for pedestrians;

$i, j, k=$ integers used for iterations $(=0,1,2, \ldots)$, or index of node, of strut or cable;

$I_{s}, I_{s, i}=$ cross-section moment of inertia of strut of index $i$;

$k=$ maximum number of struts in contact with each other;

$L=$ span of structure;

$L_{0}, L_{0, i}=$ length of member (strut or cable) of index $i$ when structure is subjected only to prestress $\tilde{\mathbf{P}}$;

$m=$ equal to ratio $e / d$;

$N_{c, i}=$ axial force in cable of index $i$, positive because always in traction, when structure is subjected to external load $\tilde{\mathbf{F}}$, prestress $\tilde{\mathbf{P}}$, and self-weight $\tilde{\mathbf{G}}$;

$N_{s, j}=$ axial force in strut of index $j$, negative because always in compression, when structure is subjected to external load $\tilde{\mathbf{F}}$, prestress $\tilde{\mathbf{P}}$, and self-weight $\tilde{\mathbf{G}}$;

$n=$ number of nodes;

$n_{c}=$ number of cables;

$n_{s}=$ number of struts;

$P, P_{i}=$ value of prestress force into cable ( $>0$ because in traction) or strut ( $<0$ because in compression) of index $i$, before application of external load;

$\mathrm{P} 1$ = family of structures composed of pentaplex of Type 1;

$\mathrm{P} 2$ = family of structures composed of pentaplex of Type 2;

$\tilde{\mathbf{P}}, \tilde{\mathbf{p}}=$ vectors defined in section "External Loads, SelfWeight, Prestress, and Security Coefficients";

Q1 = family of structures composed of quadruplex of Type 1;

Q2 = family of structures composed of quadruplex of Type 2;

$q=$ defined only for struts as $q=I_{s} / A_{s}^{2}$;

$R_{s}=$ number of support reactions;

$S=$ number of elementary tensegrity modules that compose structure;

$\mathrm{S} 1$ = family of structures composed of simplex with straight deck;

$\mathrm{S} 2$ = family of structures composed of simplex with sinusoidal deck;

$t_{i}^{F}=$ one value of vector $\tilde{\mathbf{f}}$

$t_{i}^{P}=$ one value of vector $\tilde{\mathbf{p}}$

$t_{i}^{\text {pre }}=$ one value of vector $\tilde{\mathbf{f}}_{\text {pre }}$;

$u=\sigma_{c} / \sigma_{s}$;

$V=$ volume of materials (cables and struts);

$W, W_{\min }=$ indicator of volume, equal to $\sigma_{s} V / F L$;

$\beta, \beta_{\text {pre }}=$ factor $>0$ defining prestress level;

$\beta_{\text {min }}$, = value of $\beta$ or $\beta_{\text {pre }}$ under which least-tensioned cable

$\beta_{\text {pre,min }} \quad$ slacks when loads are applied;

$\delta=$ deflection somewhere, for instance at midspan, due to live loads and self-weight acting together;

$\theta=$ real number $\geq 1$ related to prestress level;

$\Lambda_{i}=\lambda_{i} /\left(\pi \sqrt{E_{s} / \sigma_{s}}\right)$;

$\lambda_{i}=$ slenderness of strut of index $i$, equal to $L_{0, i} \sqrt{A_{s, i} / I_{s, i}}$;

$\rho=$ specific weight of material;

$\rho_{c}=$ specific weight of material used for cables;

$\rho_{s}=$ specific weight of material used for struts;

$\sigma=\sigma_{c}$ or $\sigma_{s}$

$\sigma_{c}=$ maximum allowable stress in cables (including security coefficient);

$\sigma_{s}=$ maximum allowable stress in struts (including security coefficient);

$v=$ fundamental frequency in hertz;

$\Phi=$ indicator of self-weight when $\Phi_{c}=\Phi_{s}$; 
$\Phi_{c}=\rho_{c} L / \sigma_{c}$ and $\Phi_{s}=\rho_{s} L / \sigma_{s}=$ indicators of self-weight related to cables and to struts; and

$\Psi=$ buckling indicator of structure, equal to $\sigma_{s} L /\left(q E_{s} F\right)^{1 / 2}$.

\section{References}

AASHTO. 2009. Guide specifications for design of pedestrian bridges. Washington, DC: AASHTO.

Amouri, S., J. Averseng, J. Quirant, and J.-F. Dubé. 2015. "Structural design and control of modular tensegrity structures." Eur. J. Environ. Civ. Eng. 19 (6): 687-702. https://doi.org/10.1080/19648189.2014 .965849 .

Averseng, J., and J. F. Dubé. 2012. "Design, analysis and self stress setting of a lightweight deployable tensegrity modular structure." Procedia Eng. 40: 14-19. https://doi.org/10.1016/j.proeng.2012.07.048.

Bel Hadj Ali, N., L. Rhode-Barbarigos, A. A. Pascual Albi, and I. F. C. Smith. 2010. "Design optimization and dynamic analysis of a tensegrity-based footbridge." Eng. Struct. 32 (11): 3650-3659. https:// doi.org/10.1016/j.engstruct.2010.08.009.

Briseghella, B., L. Fenu, W. Huang, and T. Zordan. 2010. "Tensegrity bridge with prestressed deck." In Proc., 34th IABSE Symp., Large Structures and Infrastructures for Environmentally Constrained and Urbanised Areas, 432. IABSE Report No. 97. Zurich, Switzerland: International Association for Bridge and Structural Engineering.

Cadoni, D., and A. Micheletti. 2012. "Structural performances of singlelayer tensegrity domes." Int. J. Space Struct. 27 (2/3): 167-178. https:// doi.org/10.1260/0266-3511.27.2-3.167.

Carpentieri, G., R. E. Skelton, and F. Fraternali. 2015. "Minimum mass and optimal complexity of planar tensegrity bridges." Int. J. Space Struct. 30 (3/4): 221-243. https://doi.org/10.1260/0266-3511.30.3-4 .221.

CEN (European Committee for Standardization). 1990. Basis of structural design, annexes A1 and A2. Eurocode 0. Brussels, Belgium: CEN.

Davey, P., and K. W. Forster. 2007. Exploring boundaries: The architecture of Wilkinson Eyre, 114-115. Berlin: De Gruyter.

De Boeck, J. 2013. "Tensegrity bridges: Concept design of pedestrian bridges using tensegrity as load carrying system.” M.Sc. thesis, Delft Univ. of Technology. https://repository.tudelft.nl/islandora/object/uuid \%3Abdab80b7-ed0e-4236-8e8f-74ff4ac75be5.

Eekhout, M. 2016. Lectures on innovation in building technology: Lecture articles for students of architecture, Delft 1992-2015/Nottingham 2005-2011. Amsterdam, Netherlands: IOS Press.

Gómez-Jáuregui, V. 2010. Tensegrity structures and their application to architecture. Santander, Cantabria, Spain: PUbliCan-Ediciones Univ. de Cantabria.

Hanaor, A. 2012. "Debunking 'tensegrity'-a personal perspective." Int. J. Space Struct. 27 (2/3): 179-183. https://doi.org/10.1260\%2F0266 $-3511.27 .2-3.179$.

Heinemeyer, C., et al. 2009. "Design of lightweight footbridges for human induced vibrations: Background document in support to the implementation, harmonization, and further development of the Eurocodes." Luxembourg: Office for Official Publications of the European Communities.

Latteur, P. 2000. Optimisation et prédimensionnement des treillis, arcs, poutres et câbles sur base d'indicateurs morphologiques. Application aux structures soumises en partie ou en totalité au flambement. Doctoral thesis, Vrije Univ. Brussel. https://orbi.uliege.be/handle/2268 130107.

Latteur, P., J. Feron, and V. Denoël. 2017. "A design methodology for lattice and tensegrity structures based on a stiffness and volume optimization algorithm using morphological indicators." Int. J. Space Struct. 32 (3/4): 226-243. https://doi.org/10.1177/0266351117746267.

Latteur, P., P. Samyn, and P. De Wilde. 2000. "Comparaison des treillis classiques de type Warren, Pratt et Howe: Optimisation et pré- dimensionnement sur base d'indicateurs morphologiques." Rev. Fr. Génie Civ. 4 (4/2000).

Latteur, P., P. Samyn, and P. De Wilde. 2001. "Optimisation des arcs funiculaires paraboliques et en chaînette: Aide à la conception sur base d'indicateurs morphologiques." Rev. Fr. Génie Civ. 5 (1): 89-116. https://doi.org/10.1080/12795119.2001.9692296.

Micheletti, A. 2012. "Modular tensegrity structures: The 'Tor Vergata' footbridge." In Mechanics, models and methods in civil engineering, edited by M. Frémond and F. Maceri, 375-384. Vol. 61 of Lecture Notes in Applied and Computational Mechanics. Berlin: Springer. https://doi.org/10.1007/978-3-642-24638-8_25.

Micheletti, A., V. Nicotra, P. Podio-Guidugli, and S. Stucchi. 2005. "The tensegrity footbridge at Torvergata University in Rome." In Proc., 2nd Int. Conf. Footbridge, 159-160. Venice: Italy.

Michell, A. G. M. 1904. "The limits of economy of material in framestructures." London, Edinburgh, Dublin Philos. Mag. J. Sci. 8 (47): 589-597. https://doi.org/10.1080/14786440409463229.

Motro, R. 2005. Tenségrité. Paris: Hermes Science.

Motro, R., B. Maurin, and C. Silvestri. 2006. "Tensegrity rings and the hollow rope." In Proc., IASS Symp. 2006: New olympics, new shells and spatial structures. 470-471. Madrid, Spain: International Association for Shell and Spatial Structures.

Mucedola, M., and S. Paradiso. 2013. "Suspended tensegrity bridge." Accessed March 23, 2018. https://issuu.com/stefanoparadiso3/docs/tesi _mucedola_paradiso.

Rhode-Barbarigos, L., N. B. H. Ali, R. Motro, and I. F. C. Smith. 2010a. "Designing tensegrity modules for pedestrian bridges." Eng. Struct. 32 (4): 1158-1167. https://doi.org/10.1016/j.engstruct.2009.12 .042 .

Rhode-Barbarigos, L., N. B. H. Ali, R. Motro, and I. F. C. Smith. 2012. "Design aspects of a deployable tensegrity-hollow-rope footbridge." Int. J. Space Struct. 27 (2/3): 81-95. https://doi.org/10.1260/0266-3511.27 .2-3.81.

Rhode-Barbarigos, L., H. Jain, P. Kripakaran, and I. F. C. Smith. 2010 b. "Design of tensegrity structures using parametric analysis and stochastic search." Eng. Comput. 26 (2): 193-203. https://doi.org/10.1007/s00366 -009-0154-1.

Rolvink, A., R. van de Straat, and J. Coenders. 2010. "Parametric structural design and beyond." Int. J. Archit. Comput. 8 (3): 319-336. https://doi .org/10.1260/1478-0771.8.3.319.

Schwartz, C., A. Berger, O. Bruls, B. Forthomme, J.-L. Croisier, and V. Denoël. 2013. "Experimental study of the human ability to deliberately excite a flexible floor." In Research and applications in structural engineering, mechanics and computation, edited by A. Zingoni, 55-58. Boca Raton, FL: CRC Press.

Sétra/AFGC. 2006. "Evaluation du comportement vibratoire des passerelles piétonnes sous l'action des piétons" [Assessment of vibrational behavior of footbridges under pedestrian loading]. Paris: Association Française de Génie Civil.

Skelton, R. E., and M. de Oliveira. 2009. Tensegrity systems. Boston: Springer. https://doi.org/10.1007/978-0-387-74242-7.

Skelton, R. E., F. Fraternali, G. Carpentieri, and A. Micheletti. 2014. "Minimum mass design of tensegrity bridges with parametric architecture and multiscale complexity." Mech. Res. Commun. 58 (Jun): 124 132. https://doi.org/10.1016/j.mechrescom.2013.10.017.

Sychterz, A. C., and I. F. C. Smith. 2017. "Joint friction during deployment of a near-full-scale tensegrity footbridge." J. Struct. Eng. 143 (9): 04017081. https://doi.org/10.1061/(ASCE)ST.1943-541X.0001817.

Van den Broeck, P., and G. De Roeck. 2012. "Tricon: Prediction and control of human-induced vibrations of civil structures." IWT Tetraproject 090137. Flanders, Belgium: Agency for Innovation by Science and Technology (IWT).

Veuve, N., S. D. Safaei, and I. F. C. Smith. 2015. "Deployment of a tensegrity footbridge." J. Struct. Eng. 141 (11): 04015021. https://doi .org/10.1061/(ASCE)ST.1943-541X.0001260.

WilkinsonEyre. 2004. "Tensegrity Bridge." Accessed September 2, 2018. http://www.wilkinsoneyre.com/projects/tensegrity-bridge. 